\title{
Light-Emitting Diodes Based on Two-Dimensional Nanoplatelets
}

\author{
Yating Guo, ${ }^{1}$ Feng Gao, ${ }^{1}$ Pan Huang, ${ }^{1}$ Rong Wu, ${ }^{1}$ Wanying Gu, ${ }^{1}$ Jing Wei $\mathbb{D}^{1},{ }^{1}$ Fangze Liu, ${ }^{2}$ \\ and Hongbo $\mathrm{Li}^{1}$
}

${ }^{1}$ Beijing Key Laboratory of Construction-Tailorable Advanced Functional, Materials and Green Applications, Experimental Center of Advanced Materials, School of Materials Science and Engineering, Beijing Institute of Technology, Beijing 100081, China

${ }^{2}$ Advanced Research Institute of Multidisciplinary Sciences, Beijing Institute of Technology, Beijing 100081, China

Correspondence should be addressed to Fangze Liu; fliu@bit.edu.cn

Received 21 October 2021; Accepted 31 December 2021; Published 7 February 2022

Copyright (c) 2022 Yating Guo et al. Exclusive Licensee Beijing Institute of Technology Press. Distributed under a Creative Commons Attribution License (CC BY 4.0).

\begin{abstract}
Colloidal semiconductor nanocrystals (NCs) attract significant interest in recent years due to their narrow and tunable emission wavelength in the visible range, as well as high photoluminescence quantum yield (PLQY), which are highly desired in display technologies. The high-quality NCs have been recognized as vital luminescent materials in realizing next-generation display devices. With further development, NCs with near-unity PLQY have been successfully synthesized through engineering of the core/shell heterostructure. However, as the external quantum efficiency (EQE) of the nanocrystal light-emitting diodes (LEDs) approaches the theoretical limit of about $20 \%$, the low out-coupling factor proposes a challenge of enhancing the performance of a device when using the spherical QDs. Hence, the anisotropic NCs like nanoplatelets (NPLs) are proposed as promising solutions to improve the performance of nanocrystal LEDs. In this review, we will summarize the synthetic strategies of twodimensional (2D) NPLs at first. Then, we will introduce fundamental concepts of LEDs, the main approaches to realize LEDs based on nanoplatelets, and the recent progress. Finally, the challenges and opportunities of LEDs based on anisotropic NCs are also presented.
\end{abstract}

\section{Introduction}

In the past few decades, colloidal nanocrystals (NCs) have drawn considerable attention due to their solution-processable and low-cost synthesis, high photoluminescence quantum yield (PLQY), and high color purity, as well as easily tunable emission wavelengths [1-4]. All these superior properties render NCs to offer great potential for light-emitting diodes (LEDs) [5-7]. The first colloidal quantum dot-based LEDs (QLEDs) were proposed in 1994, unlocking a new route to develop LEDs [8]. The device structure of QLEDs is similar to that of organic LEDs (OLEDs), except the emitting layer (EML) consists of QDs instead of organic materials (see Figure 1(a).) Over the past few years, the QLED technology has seen a huge development in the commercialization process. Compared with traditional semiconductor LEDs, the narrow emission makes QLEDs superior in presenting vibrant display with high saturation. A narrow emission will enable a wide color range display, which is attractive for television and other display fields [9-11]. Particularly, due to the saturated emission colors from QDs, QLEDs can cover the BT 2020 standard color parameters used for ultra-high-definition television systems [12-13]. Therefore, QD-based displays have received a lot more attention in solidstate lighting and display technology.

With the development of device efficiency and brightness, QLEDs also face many challenges. For example, the electrical luminescence (EL) is deteriorated when the devices are operated at high current density, and the efficiency of devices is still behind the current state-of-the-art technologies. Among many properties affecting the performance of QLEDs, the external quantum efficiency (EQE) defined by the extracted photons per injected charge is the most important and can be directly compared between different devices. The EQE consists of two parts: the internal quantum efficiency (IQE) and the out-coupling efficiency. The IQE is defined by the percentage of radiative recombination events among total recombination events or, in other words, ratio of emitted photons over injected charges. Therefore, QDs with high PLQY are more likely to reach high IQE. However, high PLQY does not necessarily guarantee high IQE since the PLQY is usually measured by optical pumping in QDs which differs from the electrical pumping in QLEDs. The 


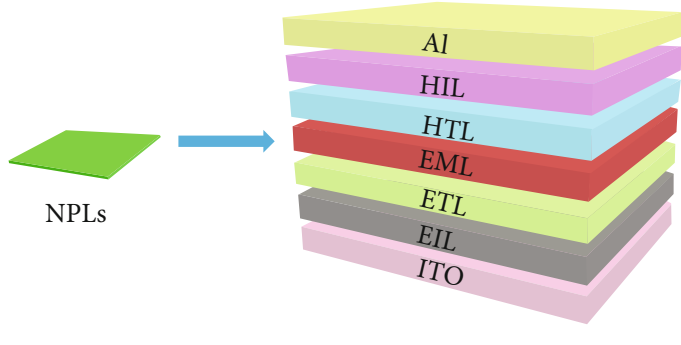

(a)

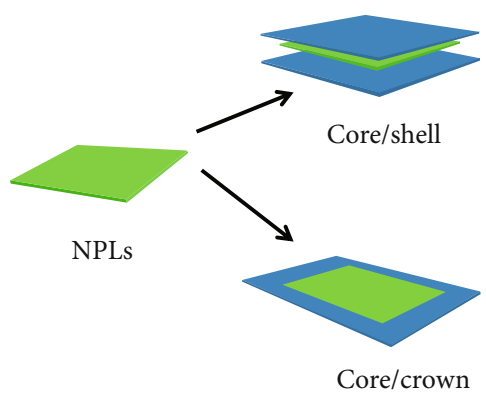

(b)

Figure 1: (a) A schematic device architecture of typical NPL-LEDs. (b) NPLs with core/shell and core/crown structure.

latter can cause unbalanced charge injection rates between electrons and holes, resulting in charged QDs and nonradiative Auger recombination [10, 14]. Though the best IQEs from QLEDs have reached near 100\%, the EQE is still limited to a maximum value of $\sim 20 \%$ mainly by the outcoupling factor, calculated by the extraction efficiency of photons from the device. Several mechanisms contribute to the loss of out-coupling factor, including photon absorption, Fresnel and total reflection, and horizontally propagating light which is generated by vertical transition dipole moments (TDM) and coupled to substrate, waveguide, and surface plasmon modes [15-16].

Several approaches have been developed to enhance the out-coupling factor, for example, structured substrate surface [17], patterning of the light-emitting layer [18], high refractive index substrate [19], and other methods that can enhance out-of-plane emission or suppress total internal reflection [20-23]. Due to the randomly oriented TDMs, the out-coupling factor of QLEDs based on spherical QDs is most limited by the transverse magnetic (TM) mode generated by vertical TDMs. Therefore, anisotropic semiconductor nanocrystals (aNCs) like nanoplatelets (NPLs) are utilized as emitters in LEDs to address the current difficulties. Unlike spherical QDs, aNCs exhibit strong inplane TDMs that can double the out-coupling factor (from 0.2 to 0.4$)[16,24-25]$. aNCs also have many outstanding optical and physical properties that are different from zero-dimensional (0D) QDs. The strong quantum confinement in the vertical direction of NPLs leads to thicknessdependent optical properties, for example, narrow emission, large exciton binding energy, relatively small dielectric constant, giant oscillator strength, extremely large linear/nonlinear absorption cross sections, and ultrashort radiative fluorescence lifetime [26-30]. Additionally, because of the nanostructures with an extended volume, the Auger recombination becomes unlikely. In other word, the Auger recombination of charged excitons in NPLs is suppressed by their anisotropic geometry [31-33]. All of these advantages make aNCs promising for next-generation LEDs.

Significant progress has been made for LEDs based on aNCs during the past few years. They can be mainly divided into two categories, namely, CdSe-based and perovskitebased aNCs. To improve the performance of the LEDs based on aNCs, lots of efforts have been made to material explora- tion, device physics, and structure optimization. The NPLs with different heterostructures are raised to optimize the LED performance (such as core/shell and core/crown, Figure 1(b)). In this review, we will first present the fundamental concepts of LEDs. Then, we will introduce the synthetic strategies of NPLs. Next, we will summarize two main types of NPL-LEDs, including CdSe and perovskite materials. More specifically, we will focus on the design strategy, architecture of NPL-LEDs, and methods to enhance performance. Finally, we will overview the challenges and perspectives of NPL-LEDs.

\section{Fundamentals of LEDs Based on Anisotropic Nanocrystals}

Compared with QLED or OLED, the development of LEDs based on aNCs is still at an early stage. However, these technologies show many similarities in device structures as well as working mechanisms. For example, the emitting layer (EML) of aNC-LED is sandwiched between the electron transport layer (ETL) and the hole transport layer (HTL), which is similar to QLED and OLED. Different from QLED or OLED, aNC-LEDs use NPLs as the emitters for their outstanding properties like narrow emission spectrum and large surface area. In terms of the ETL and HTL, their main function is to ensure that holes/electrons can efficiently transport to the EML. In order to confine the electrons within the EML and reduce the leakage electron flow, the ETL with the shallow lowest unoccupied molecular orbital (LUMO) or conduction band minimum (CBM) and HTL with deep highest occupied molecular orbital (HOMO) or valence band maximum (VBM) are desired [34-37]. Furthermore, a hole injection layer (HIL)/electron injection layer (EIL) is used to ensure that holes/electrons can be effectively injected into the HTL/ETL from the anode/cathode. In particular, $\mathrm{ZnO}$ nanoparticles (NPs) are wildly used as ETL because of their high mobility $\left(\sim 1.3 \times 10^{-3} \mathrm{~cm}^{2} \mathrm{~V}^{-1} \mathrm{~s}^{-1}\right)$, simple synthesis, and suitable $\mathrm{CBM}$ of $4.4 \mathrm{eV}$ which matches the work function of the commonly used indium tin oxide (ITO, $\sim 4.7 \mathrm{eV}$ ) cathode [38].

The emission mechanism needs to be well understood in NPL-LEDs to improve their performance. The LED performance is commonly evaluated by several parameters, including EQE, power efficiency (PE), and emission color. The EQE can be expressed as 


$$
\mathrm{EQE}=\eta_{\text {out }} \mathrm{IQE}=\eta_{\text {out }}(r \gamma q),
$$

where $\mathrm{EQE}$ is defined as a ratio of the number of photons emitted out of an LED to the number of injected electrons, IQE is the internal quantum efficiency, $\eta_{\text {out }}$ is the outcoupling efficiency calculated by the fraction of photons escaping out of the device, $r$ is the fraction of excitons that radiatively decay, $\gamma$ represents the charge balance, and $q$ represents the radiative recombination efficiency of the emitter. $\eta_{\text {out }}$ is restricted by the total internal reflection due to the large difference in refraction indices of the different layers in LEDs. For most QLED, the $\eta_{\text {out }}$ is normally limited to 0.2 which further restricts the EQE to $\sim 20 \%$ [3, 14, 39-41]. Several strategies have been developed to solve this issue, such as microlens array, surface plasmon resonance, and surface coarsening. However, due to the random TDM orientation in spherical QDs, QLEDs show a fixed value of the out-coupling efficiency at 0.2. Compared with QLEDs, NPL-LEDs show doubled out-coupling efficiency because of the anisotropic geometry of NPLs [42].

In addition to the light out-coupling efficiency, the IQE also needs to be increased to achieve highly efficient NPL-LEDs. A straight way to increase the IQE is to generate more photons in the NPLs by suppressing nonradiative exciton recombination. Hence, NPLs with core/shell, core/ crown, or alloyed core structures are proposed. Besides, heterostructure NPLs with high PLQY and low surface defect densities are beneficial to fabricate high-quality LEDs. Controlling the shell thickness can also reduce the Auger process [43].

Furthermore, because unfavorable energy barrier exists between adjancet layers in LEDs, enhancing the charge balance is also critical to improve the performance of LEDs. Therefore, charge transport materials are the key to charge balance. There are four categories of charge transport materials used in QD-LEDs, including polymer, organic small molecule, inorganic and hybrid organic-inorganic charge transport materials $[1,44-46]$. The recent emergence of organic-inorganic hybrid charge transport materials has led to the rapid development of QLEDs. For NPL-LEDs, the selections of charge transport materials are still limited. Hence, many efforts are urgently needed to improve the charge balance in NPL-LEDs. Several strategies have been reported, such as doping small amount of PVK (5 wt\%) into EML [47] and introducing a PMMA layer between EML and HTL [3].

The PE can be calculated in terms of EQE and the voltage $U$ applied to the LED: [48]

$$
\mathrm{PE}=\frac{\overline{\hbar \omega}}{q U} \mathrm{EQE},
$$

where $\overline{\hbar \omega}$ is the average energy of emitted photons and $q$ is the elementary charge. Obviously, high $\mathrm{EQE}$ and low $U$ result in high PE where low $U$ can be acquired through applying materials with high charge mobility or reducing the energy barriers between adjacent layers [49-50]. Finally, the emission color determines the color gamut of displays made of NPL-LEDs which can be measured by the CIE (Commission Internationale de l'Eclairage) color coordinates. Specifically, narrower emission spectrum leads to higher color space [51]. Different from colloidal QDs which need to strictly control three dimensions for narrow spectrum, NPLs only need to precisely control the thickness. The heterostructures of NPLs with uniform thickness exhibit narrow full width at half maximum (FWHM) $(\sim 20 \mathrm{~nm})$ which is highly beneficial to the color purity.

\section{The Synthetic Strategies of 2D Nanoplatelets}

Since the early 2000s, the anisotropic growth of NCs was first achieved for nanorods and tetrapods [52-53]. Later, it was found that the right choice of ligands could control the shape of NCs from spherical seeds to 2D NPLs [52-56]. Since the CdSe NPLs were first reported in 2008 [54], the 2D NPLs quickly received intense interest, and different strategies were developed to systematically tune the thickness, shape, and doping of NPLs [26, 57]. Heterostructured NPLs including core/shell and core/crown were also developed [30, 58-59]. During the past few years, perovskite NPLs have attracted a lot of attention. The organicinorganic perovskite $\mathrm{MAPbBr}_{3}$ (methylammonium (MA)) NPLs were first synthesized by Tyagi et al. in 2015 [60]. The inorganic perovskite $\mathrm{CsPbBr}_{3}$ NPLs with high PLQY (up to 84\%) were first reported by Bekenstein et al. [61] By cation exchange, $\mathrm{CsPbI}_{3}$ and $\mathrm{CsPbCl}$ nanoplatelets were also prepared. In recent years, the synthesis of NPLs with thickness tunability, shape control, heterostructures, and controlled doping has made great progress.

3.1. Thickness Tunability. The optical properties of NPLs are in close relation to the thickness which is defined as the number of monolayers (MLs). Typically, the optical gain threshold and Auger recombination rate are strongly correlated with the thickness of NPLs [4, 33, 62]. It has been reported that CdSe NPLs emitting at 390-550 nm, corresponding to thickness of 2-5 ML, can be synthesized by modifying the growth temperature and corresponding seed diameter [6, 54-55, 63]. Ithurria et al. studied the formation process of CdSe NPLs through a combination of transmission electron microscopy (TEM) and absorption/emission spectroscopy [55]. It was found that the growth of NPLs started from $\sim 2 \mathrm{~nm}$ diameter seeds and continued by the lateral extension to form NPLs. The thickness of NPLs was determined by the seed dimension and remained unchanged during the growth. By slightly modifying the growth condition to change the size of seeds, CdSe NPLs can be grown with controlled thickness $(3,4$, and $5 \mathrm{ML})$ and lateral dimension of tens of nanometers. The growth mechanism of NPLs was further studied by Riedinger et al. [64] By combining experimental and theoretical studies, it was confirmed that the growth of NPLs was mainly driven by the much lower energy required to grow on the side facets than that to create a top or bottom layer, resulting in a very large difference in growth rate between lateral and vertical directions. However, due to the difficulty for larger seeds to grow into anisotropic 


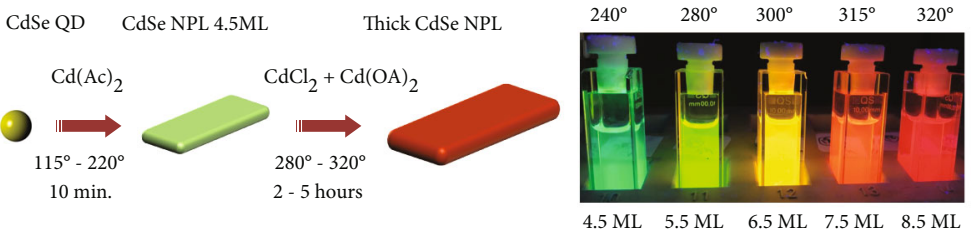

(a)

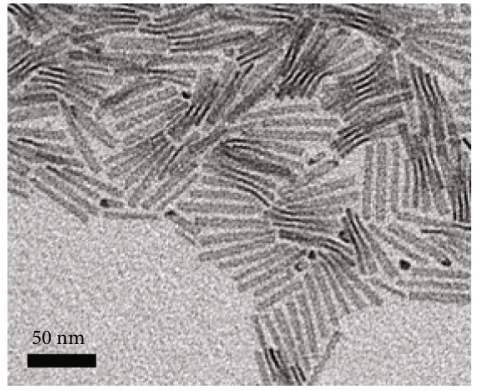

(c)

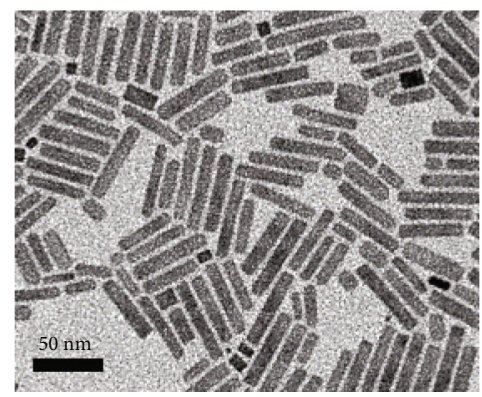

(d)

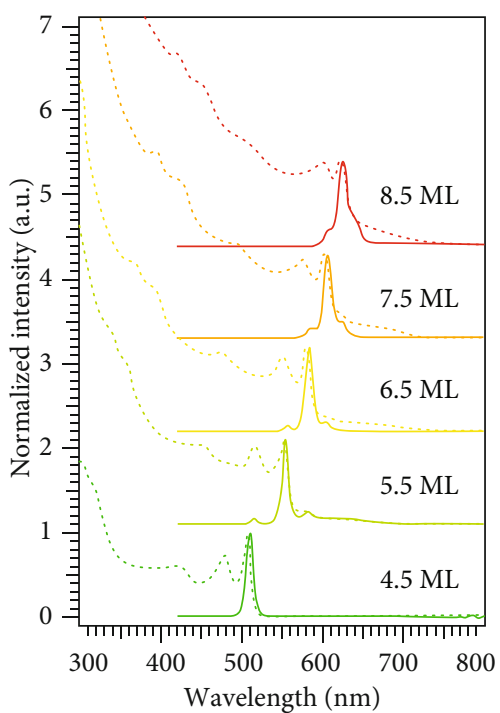

(b)

FIgURE 2: (a) Reaction schematic, with a photograph of the different NPL samples under UV illumination. (b) Absorption and PL spectra of the NPL series. (c, d) BF-TEM images of 4.5 ML and 7.5 ML CdSe NPLs. Adapted with permission from reference [65]. Copyright 2018 American Chemical Society.

NPLs, little work has been done for direct synthesis of thick CdSe NPLs. Christodoulou et al. used a two-step reaction that switched from 2D to 3D growth and obtained thick (8.5 ML) CdSe NPLs with emission peak at $625 \mathrm{~nm}$ (Figure 2(b)) [65]. In this work, 4.5 ML CdSe NPLs were first synthesized; then, the temperature was increased to 280$320^{\circ} \mathrm{C}$ and chloride $\left(\mathrm{CdCl}_{2}\right)$ was introduced to stimulate the growth along the (001) direction on the top and bottom surface of CdSe NPLs to obtain the final thick NPLs (Figure 2(a)). The TEM images are shown in Figures 2(c) and 2(d). As reported by Cho et al., $\mathrm{Cl}^{-}$anions on the NPL can not only modify the surface energy but also reduce the nucleation barrier of CdSe islands on the basal facets [66]. Hence, the vertical growth rate was increased, resulting in more isotropic growth. This work provided a new pathway to modulate the thickness of NPLs.

For inorganic perovskite NPLs, it has been shown that $\mathrm{CsPbBr}_{3}$ NPLs were prepared with thickness in the range of 1 to 5 MLs by varying reaction temperature $\left(90-130^{\circ} \mathrm{C}\right)$ [62]. The same materials have also been reported by Akkerman et al. $[62,65] \mathrm{CsPbBr}_{3}$ NPLs can form in seconds by injecting acetone into the mixture of Cs oleate, $\mathrm{PbBr}_{2}, \mathrm{HBr}$ oleylamine, and oleic acid. The thickness of NPLs can be controlled by the amount of $\mathrm{HBr}$ in the mixture. Furthermore, Zhao et al. reported $\mathrm{CsPbBr}_{3}$ NPLs synthesized by an amino acid-mediated pathway with thickness from 4 to 12 ML by varying tryptophan (Trp) concentration [67]. The growth mechanism was proposed as the nitrogen in the indole ring of Trp attached on $\mathrm{Pb}^{2+}$ restraining the growth in the vertical direction, leading to the formation of NPLs. For organic-inorganic perovskite NPLs, thickness tunability and consequent changes in optical properties have also been studied. Weidman et al. synthesized $n=1$ and $n=2$ NPLs in the form of $\mathrm{L}_{2}\left[\mathrm{ABX}_{3}\right]_{n-1} \mathrm{BX}_{4}$, where $n$ represented the layers of metal halide octahedral and was controlled by the proportion of precursor salts $\left(\mathrm{AX}, \mathrm{BX}_{2}\right.$, and $\left.\mathrm{LX}\right)$ added [68]. It was observed that $n=1$ NPL had reduced PLQY compared to $n=2$ NPLs, which was in agreement with the thickness-dependent trend.

3.2. Shape Control. Similar to the thickness tunability, the shape and area control of NPLs are also important methods to tune the properties of NPLs. The control of the NPL aspect ratio (AR) can be achieved by modifying the proportion of hydrated $\mathrm{Cd}(\mathrm{OAc})_{2}$ in a $\mathrm{Cd}(\mathrm{OAc})_{2} / \mathrm{Cd}$ $(\mathrm{OAc})_{2} \cdot 2 \mathrm{H}_{2} \mathrm{O}$ mixture [63]. It was shown that the $\mathrm{AR}$ of CdSe NPLs depends on the formation of hydroxide anions in the cadmium-acetate precursor. The CdSe NPLs can be tuned from square (AR $1: 1)$ with a dry $\mathrm{Cd}(\mathrm{OAC})_{2}$ precursor to elongated rectangles (AR 7.7:1) with a mixture of 30 $\mathrm{mol} \%$ dry $\mathrm{Cd}(\mathrm{OAc})_{2}$ and $70 \mathrm{~mol} \% \mathrm{Cd}(\mathrm{OAc})_{2} \cdot 2 \mathrm{H}_{2} \mathrm{O}$ precursor. It was speculated that the surface energy balance can be adsorbed and altered via hydroxide anion, leading to different shapes through varying the growth rate of specific facets. This work demonstrated that the $\mathrm{OH}^{-}$is crucial to controlling the shape of NPLs in the growth.

Halides have been traditionally used in the synthesis of NCs to modify the shape, dimension, phase, etc. [69-70] Gerdes et al. added different amounts of halogenated alkanes (1-bromoheptane (Hep), 0.0625 to $4 \mathrm{mmol}$ ) to the hotinjection synthesis to tune the CdSe NPLs' shape from sexangular to quadrangular and triangular [71]. The in situformed halide ions in 1-bromoheptane worked as cadmium complexing agents and surface X-type ligands to alter the nucleation and growth to change the shape of NPLs. This work also proved that different amounts of Cl-Hep and IHep also control the shape of NPLs. Moreover, high concentrations of Br-Hep can change the phase of NPLs from zinc blende (ZB) to wurtzite (WZ). 
Apart from the well-developed metal chalcogenide NCs, shape control of perovskite NCs has also been increasingly studied in recent years. Liang et al. first reported a shapecontrolled synthesis of all-inorganic $\mathrm{Cs}_{\mathrm{PbBr}}$ perovskite $\mathrm{NCs}$ [72]. Shapes of the prepared $\mathrm{Cs} \mathrm{PbBr}_{3} \mathrm{NCs}$ could be systematically engineered into single and lamellar-structured 0D quantum dots, as well as face-to-face stacking 2D NPLs and flat-lying 2D nanosheets via tuning the amounts of oleic acid (OA) and oleylamine (OM). The $\mathrm{CsPbBr}_{3}$ NPLs obtained by adding $0.5 \mathrm{~mL}$ of OA and $0.5 \mathrm{~mL}$ of $\mathrm{OM}$ showed the PL emission peak of $449 \mathrm{~nm}$. This work was significant for the application of inorganic perovskite in blue LEDs.

3.3. Heterostructures. Due to the outstanding optical features, such as narrow FWHM of PL spectrum and fast PL decay, NPLs have become promising candidates for nextgeneration quantum emitters [73]. For this purpose, increasing further their PLQY is critical. For QDs, the widely accepted strategy is in situ growth of heterostructures where the exciton wave function can be confined within the core by type I or quasi-type II band alignment between the core and the shell [74-75]. This can further reduce the effect of surface defects. Consequently, the PLQY and PL stability can be significantly increased. In this section, we will discuss the heterostructures of 2D NPLs including core/shell and core/crown heterostructures.

3.3.1. Core/Shell Structure. Currently, cadmium chalcogenide NPLs are still arousing the interest of many researchers due to their excellent optical performance. Table 1 summarizes the properties of several CdSe-based core/shell NPLs. Early studies often use low-temperature routes to grow NPL heterostructures due to the instability of NPLs under high temperature. Mahler et al. first reported mixing CdSe NPLs with thioacetamide (TAA), cadmium nitrate, octylamine, and chloroform to prepare the core/shell structure [30]. During the reaction, TAA and octylamine formed an ammonium sulfide complex in situ which acted as the sulfur precursor. The growth was carried out for several hours at room temperature to grow 3 to $5 \mathrm{ML} \mathrm{CdZnS} \mathrm{shells.} \mathrm{Polovitsyn} \mathrm{et} \mathrm{al.}$ reported a single-source precursor method based on zinc diethyldithiocarbamate (Zn (DDTC) ${ }_{2}$ ) [76]. CdSe NPLs in hexane were mixed in toluene, followed by adding a mixture of $\mathrm{ZnCl}_{2}$ in oleylamine, N-trioctylphosphine (TOP), $\mathrm{Zn}$ $(\mathrm{DDTC})_{2}$, and carbon disulfide $\left(\mathrm{CS}_{2}\right)$. The reaction finished after $1 \mathrm{~h}$ at $110^{\circ} \mathrm{C}$ to grow the $\mathrm{ZnS}$ shell. By varying the amounts of injected precursors, a $12 \mathrm{ML} \mathrm{ZnS}$ shell can be obtained. Using 1.2-dichlorobenzene as solvent, precursors were injected by a syringe pump at $150^{\circ} \mathrm{C}$ and a thicker $\mathrm{ZnS}$ shell can be obtained. CdSe/CdS and CdSe/CdZnS nanoplatelets can be prepared by the same procedure.

Another low-temperature method for shell growth is the colloidal atomic layer deposition (c-ALD) [58]. In this procedure, the reaction is conducted in polar medium, and the sulfide source often uses the $\mathrm{S}^{2-}$ precursor. The growth depends on the phase transfer of the NPLs from a nonpolar phase to a polar phase and the ligand change from oleate to $\mathrm{S}^{2-}$ or $\mathrm{SH}^{-}$. The ligands can then continuously react with the NPL surface through adding $\mathrm{Cd}$ and $\mathrm{S}$ precursors. This
TABLE 1: Summary of the typical properties of core/shell NPLs.

\begin{tabular}{|c|c|c|c|c|}
\hline Chemical composition & $\begin{array}{l}\text { Emission peak } \\
(\mathrm{nm})\end{array}$ & $\begin{array}{l}\text { FWHM } \\
(\mathrm{nm})\end{array}$ & $\begin{array}{l}\text { PLQY } \\
(\%)\end{array}$ & $\operatorname{Re}$ \\
\hline $\mathrm{CdSe} / \mathrm{CdS}$ & 586 & $\sim 20$ & & 30 \\
\hline $\mathrm{CdSe} / \mathrm{Cd}_{0.7} \mathrm{Zn}_{0.3} \mathrm{~S}$ & 644 & & 60 & 30 \\
\hline $\mathrm{CdSe} / \mathrm{ZnS}$ & $593-611$ & $20-23$ & $58 \sim 64$ & 76 \\
\hline $\mathrm{CdSe} / \mathrm{CdZnS}$ & 633 & $\sim 20$ & 80 & 78 \\
\hline $\mathrm{CdSe} / \mathrm{CdS}$ & 667 & 20 & $60 \sim 70$ & 79 \\
\hline $\mathrm{CdSe} / \mathrm{ZnS}$ & 616 & 27 & 98 & 80 \\
\hline $\mathrm{CdSe} / \mathrm{CdS} / \mathrm{ZnS}$ & 662 & 23.6 & 78 & 81 \\
\hline 4-ML-CdSe/Cd $\mathrm{Zn}_{1-x} \mathrm{~S}$ & 655 & 19.5 & 88 & 81 \\
\hline 6-ML-CdSe/Cd $\mathrm{Zn}_{1-x} \mathrm{~S}$ & 692 & 22.1 & 92 & 81 \\
\hline $\mathrm{CdSe} / \mathrm{ZnSe}$ & 684 & 22.1 & 47 & 81 \\
\hline
\end{tabular}

method can also tune the properties of charge carriers which are critical for narrow band gap thin-film transistors [77].

Though the low-temperature growth of the shell can enhance the optical performance of NPLs, the PLQY is still relatively low and the FWHM is often broadened due to the nonuniform and defective growth at low temperature $[30,76,78]$. Thus, high-temperature shell growth was proposed to overcome these limitations. Rossinelli et al. reported the growth of uniform CdS shells over CdSe nanoplatelets at $300^{\circ} \mathrm{C}$ [79]. CdSe NPLs, Cd(oleate) $)_{2}$, and 1octadecene (ODE) were first mixed in a round-bottom flask and heated to $80^{\circ} \mathrm{C}$ when oleylamine $(\mathrm{OAm})$ was added. The temperature was raised to $300^{\circ} \mathrm{C}$ under $\mathrm{N}_{2}$. Next, 1octanethiol in ODE as the $\mathrm{S}$ precursor was injected at a rate of $3 \mathrm{ml} / \mathrm{h}$ to prevent the formation of shells with irregular shapes. Finally, CdSe/CdS core/shell NPLs with emission at $667 \mathrm{~nm}$ and a narrow FWHM of $20 \mathrm{~nm}$ were obtained. It was noted that although OAm can etch CdSe NPLs, it can also increase the stability and PLQY of NPLs by passivation and stabilization of surface facets. The increased PL linewidth $(20 \mathrm{~nm})$ of core/shell NPL ensemble compared with that of core CdSe NPLs was attributed to the phonon coupling caused by the strain at the interface or the nonuniform shell thickness. The optimal reaction temperature was also identified at $300^{\circ} \mathrm{C}$. This method overcomed the disadvantages of low-temperature growth to get high-quality products with higher PLQY and stability. Similar methods were also applied to grow CdSe/ZnS [80], CdSe/ZnSe, CdSe/ $\mathrm{ZnCdS}$, and CdSe/CdS/ZnCdS core/shell NPLs [81]. Altintas et al. used a modified recipe from the previous report [79] for hot-injection growth of the $\mathrm{ZnS}$ shell for CdSe/ZnS core/shell NPLs [80]. Zn-acetate and octanethiol were selected as $\mathrm{Zn}$ and $\mathrm{S}$ precursors, respectively. A high temperature of $573 \mathrm{~K}$ was required for the shell growth due to the low reactivity of octanethiol. Normalized PL and absorption spectra for CdSe/ZnS NPLs with different shell growth time (Figure 3(a)) revealed redshifted excitonic features as the thickness of the $\mathrm{ZnS}$ shell increases. With optimized ligand concentrations (OA and ODE), the thick-shell CdSe/ZnS NPLs exhibited near-unity PLQY of 98\% (Figures 3(b) and $3(\mathrm{c})$ ). Therefore, for shell growth, high temperature is considered a better strategy than low temperature for bright NPLs. 


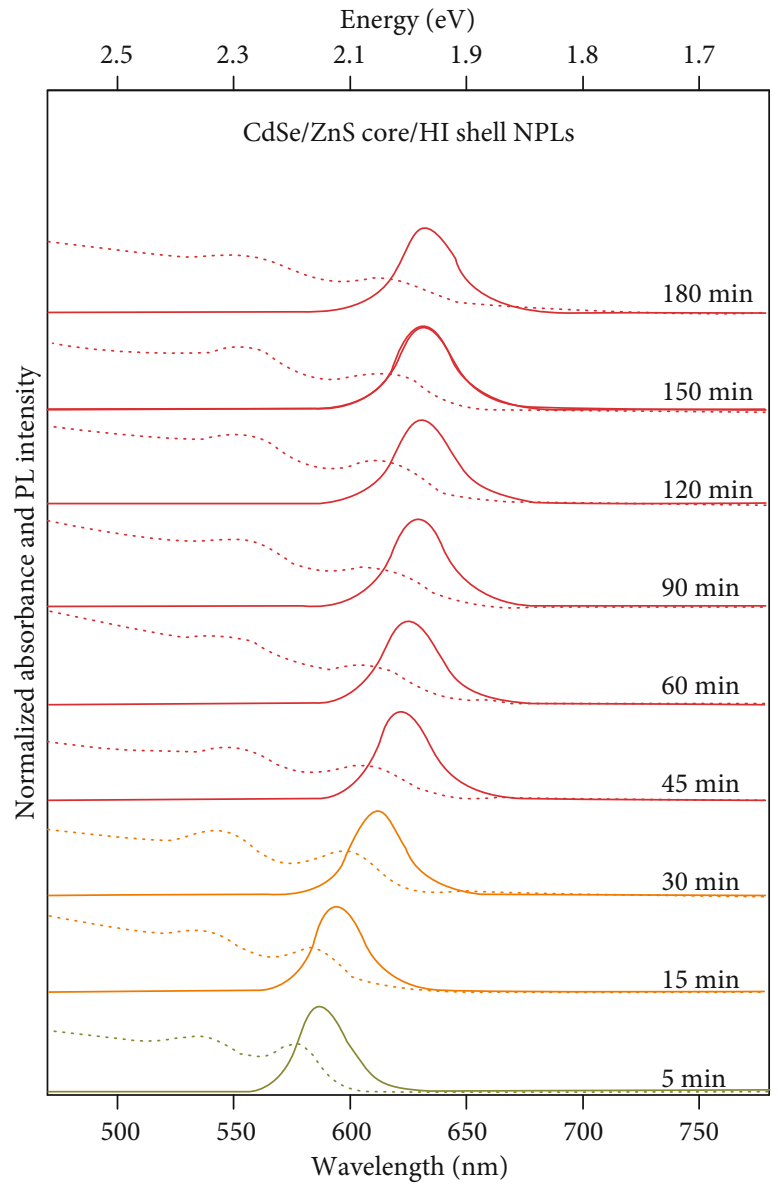

ABS

PL

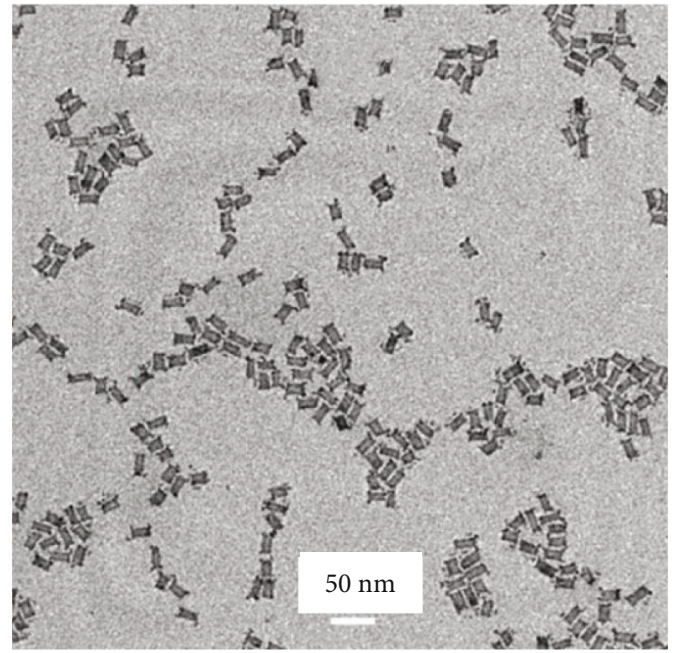

(b)

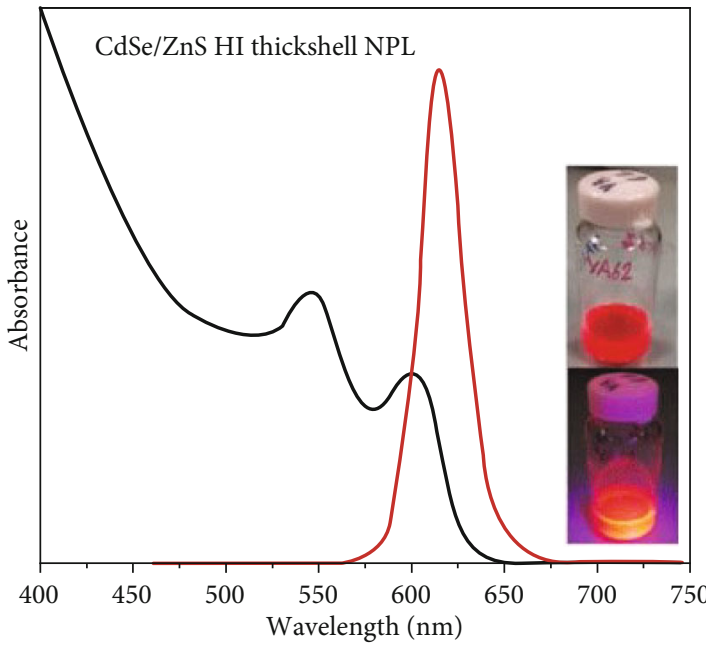

Absorbance PL intensity

(a)

(c)

Figure 3: (a) Absorption and emission spectra of CdSe/ZnS core/shell NPLs during the reaction showed redshift as the shell was growing thicker. (b, c) TEM image (b) of thick-shell CdSe ZnS NPLs with 98\% PLQY (c). Adapted with permission from reference [80]. Copyright 2019 Wiley-VCH GmbH.

3.3.2. Core/Crown Structure. Different from the core/shell structure where the growth of the shell occurs over all of the facets of the NPLs, the growth of the crown in the core-crown structure occurs only in the plane direction. In a typical reaction, the crown growth precursor was mixed with the $\mathrm{Cd}$ precursor $\left(\mathrm{Cd}(\mathrm{OAc}) 2 \cdot 2 \mathrm{H}_{2} \mathrm{O}\right.$ and oleic acid) and $\mathrm{S}$ precursor (S-ODE or sulfur in octadecene). The mixture was then injected into the CdSe NPLs dispersed in ODE. The lateral extension of the crown can be modified by the amount of the introduced precursor. CdSe/CdS core/crown NPLs have shown high PLQY while the narrow FWHM (less than $15 \mathrm{~nm}$ ) remained [28].

The core/crown structure has been extended to type II band alignment with a crown made of CdTe [59]. The
TOP-Te was used as the Te precursor. The electrons and holes are spatially separated between the core and the crown, and redshifted PL emission can be observed. Recently, a ternary heterostructure of $\mathrm{CdSe} / \mathrm{CdS} / \mathrm{CdTe}$ core/barrier/crown nanoplatelets was reported [82]. Here, CdSe NPL is a core, $\mathrm{CdS}$ is a type I barrier, and CdTe is a crown. The CdS precursor was first prepared by mixing ODE-S, Cd $(\mathrm{OAc})_{2} \cdot 2 \mathrm{H}_{2} \mathrm{O}$, ODE, and oleic acid. CdSe NPLs, ODE, and $\mathrm{Cd}$ (propionate) $)_{2}$ were mixed and heated to $235^{\circ} \mathrm{C}$ when $\mathrm{CdS}$ growth solution and Te growth solution were added dropwise $(3 \mathrm{ml} / \mathrm{h})$ and react separately for a certain time. The CdSe/CdS/CdTe heterostructure allowed adjusting both direct and indirect transitions and even photon upconversions by tuning the barrier and crown thickness. 
3.4. Doping. At present, the thickness of 2D NPLs has been perfectly controlled, and as a result, the inhomogeneous broadening of the optical features does not emerge. However, the continuous tunability of the PL emission energy is lost due to the stepwise growth. It has been demonstrated that doping is a feasible method to tune the emission color from green to red for NPLs. Sharma et al. demonstrated that introducing copper ions into CdSe NPLs led to a large Stokes shift of the PL energy from 640 to $830 \mathrm{~nm}$ [83].

Cation exchange has been proposed as a favorable choice for doping 2D NPLs [84]. In cation exchange reactions, host cations in the crystal lattice are replaced by minority dopant cations, resulting in interesting optical properties. Dufour et al. used cation exchange to prepare $\mathrm{Ag}^{+}$-doped $\mathrm{CdSe}$ NPLs (see Figures 4(a)-4(c)) [85]. $\mathrm{Ag}^{+}$cations were induced by adding silver oleate dissolved in hexane to the solution of CdSe NPLs at a rate of $6 \mathrm{ml} / \mathrm{h}$. The TEM images of doped NPLs are shown in Figures 4(d)-4(f). The absorption spectrum remained unaffected after $\mathrm{Ag}^{+}$doping, while the PL spectrum changed significantly. Without $\mathrm{Ag}^{+}$cations, CdSe NPLs showed a narrow emission at $510 \mathrm{~nm}$ with FWHM of $8 \mathrm{~nm}$. However, after $\mathrm{Ag}^{+}$doping, a broad $(\mathrm{FWHM}=18 \mathrm{~nm})$ as well as redshifted emission at $660 \mathrm{~nm}$ appeared (see Figures 4(g) and 4(h)). The emission color of NPLs can be tuned from green to yellow, orange, or red by tuning the amount of $\mathrm{Ag}^{+}$cations (see Figure 4(j)). With the continuous tunability of the emission color, this material can be applied for display applications.

Khan et al. used the same method to obtain colloidal Agdoped CdSe NPLs [86]. The emission from 609 to $880 \mathrm{~nm}$ can be achieved by doping with $\mathrm{Ag}^{+}$. They demonstrated that lifetimes of CdSe:Ag NPLs were about twice longer than those of CdSe:Ag QDs (200 ns), and the electronhole overlap was less than that of QDs. In addition, these CdSe: Ag NPLs had a strongly suppressed emission reabsorption due to the large Stokes shift. Different from the previous reports that reacted at or below room temperature, Galle et al. reported a seeded-growth method by injecting solutions of cadmium acetate dihydrate, selenium dioxide, and mercury acetate [87]. This reaction took place at high temperature $\left(200^{\circ} \mathrm{C}\right.$ or $\left.240^{\circ} \mathrm{C}\right)$, and the temperature played an important role in controlling the incorporation of the $\mathrm{Hg}^{+}$ions; i.e., the emitters showed two red $\mathrm{PL}$ emission peaks at $200^{\circ} \mathrm{C}$, while only one can be observed at $240^{\circ} \mathrm{C}$.

Doping of NPL heterostructures have also been observed. Delikanli et al. reported the synthesis of $\mathrm{Mn}^{2+}$ doped CdSe/CdS core/shell and core/multishell NPL heterostructures [88]. They grew a $\mathrm{Cd}_{0.985} \mathrm{Mn}_{0.015} \mathrm{~S}$ monolayer shell on undoped CdSe NPLs using the c-ALD technique. The locations of the Mn ions were controlled with atomic layer precision, which contributed to a narrow photoluminescence bandwidth of $\sim 22 \mathrm{~nm}$.

2D perovskite NPLs also exhibit interesting properties after doping. Mir et al. prepared $2.2 \mathrm{~nm}$ thick Mn-doped $\mathrm{CsPbCl}_{3} \mathrm{NPLs}$ [89]. A solution of Cs-oleate, ODE, OA, and OAM was vigorously stirred at first. Next, a mixture of $\mathrm{PbCl}_{2}$ solution $\left(\mathrm{PbCl}_{2}, \mathrm{DMSO}, \mathrm{DMF}, \mathrm{HCl}\right.$, and $\left.\mathrm{HNO}_{3}\right)$ and $\mathrm{MnCl}_{2} \cdot 4 \mathrm{H}_{2} \mathrm{O}$ was injected to grow $\mathrm{Mn}$-doped $\mathrm{CsPbCl}_{3}$ NPLs. The excitonic energy can efficiently transfer to the
$\mathrm{Mn}^{2+}$ dopants emitting orange PL from the $d$ electrons. In addition, due to the spin-forbidden ${ }_{4} \mathrm{~T}^{1}-{ }_{6} \mathrm{~A}^{1}$ transition of the $\mathrm{Mn} d$ electron, the PL showed a long lifetime of 1.6 ms. Mn-doped $\mathrm{CsPbCl}_{3}$ NPLs were further converted into Mn-doped $\mathrm{CsPbBr}_{3}$ NPLs through anion exchange with a weaker Mn emission. Subsequently, Li et al. proposed a facile solvothermal method which can efficiently dope $\mathrm{CsPbCl}_{3}$ NPLs with $\mathrm{Mn}$. Mn could even completely replace $\mathrm{Pb}$ to form a new $\mathrm{CsMnCl}_{3}$ phase by spinodal decomposition, while the host perovskite NPLs had no morphology or size change [90]. This work provided a new path to study interactions between dopant and NCs. Very recently, Davis et al. demonstrated an extremely facile ligand-mediated synthesis of $2 \mathrm{D} \mathrm{CsPbX}{ }_{3}$ NPLs under ambient conditions by controlling the surface ligand composition and concentration of a mixture of short (8-carbon chain) and long (18-carbon chain) ligands [91]. This method was also applied to the synthesis of $\mathrm{Mn}: \mathrm{CsPbX}_{3}$ NPLs. Furthermore, the effect of $\mathrm{Mn}^{2+}$ ion doping on the charge carrier dynamics of $\mathrm{CsPbBr}_{3} \mathrm{NPLs}$ has been explored by Babu et al. [92] They found that Mndoped $\mathrm{CsPbBr}_{3}$ NPLs exhibited utterly fast, concurrent charge (electron) and energy transfer from the host to the dopant.

\section{LEDs Based on Nanoplatelets}

NPL-LEDs can be generally divided into three categories by the EML materials, namely, CdSe, all-inorganic perovskite, and hybrid perovskite NPL-LEDs. By the device architecture, these LEDs can also be grouped into two categories, i.e., conventional and inverted structures. In conventional structure, the HIL and HTL are at the bottom of the device and in direct contact to ITO while in inverted structure, the EIL and ETL are at the bottom. Both conventional and inverted structures can be applied for NPL-LEDs, and the choice of device structures is determined by the combination of material compatibility, device fabrication process, and electron/hole injection efficiency.

\subsection{CdSe-Based NPL-LEDs}

4.1.1. Core-Only CdSe NPL-LEDs. In 2015, Vitukhnovsky et al. reported core-only CdSe NPL-LEDs [93]. They used CdSe NPLs as emitters and organic 3-(biphenyl-4-yl)-5-(4tert-butylphenyl)-4-phenyl-4H-1,2,4-triazole (TAZ) and $\mathrm{N}, \mathrm{N}^{\prime}$-bis (3-methylphenyl)-N, $\mathrm{N}^{\prime}$-bis(phenyl)-benzidine (TPD) materials as the electron and hole transporting layers for NPL-LEDs. Since core-only NPLs have narrower PL emission than core/shell NPLs, the former can potentially offer NPL-LEDs with better color purity. In this work, the NPL-LED can exhibit an EL emission peak at $515 \mathrm{~nm}$ under a turn-on voltage of $5.5 \mathrm{~V}$. However, compared with the PL spectrum of CdSe NPLs, the EL spectrum was significantly broadened, possibly because of the change in position of luminescence maxima of CdSe NPLs located in the vicinity of electron and hole transporting layers.

Fan et al. also demonstrated NPL-LEDs based on alloyed $\mathrm{CdSe}_{1-x} \mathrm{~S}_{x}$ NPLs [7]. These alloyed NPLs showed uniform lateral dimensions and thickness of 5 to $6 \mathrm{ML}$, and the PL 


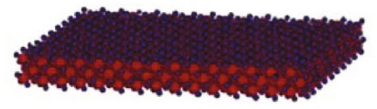

(a)

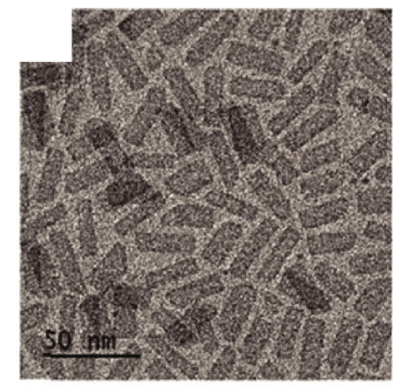

(d)
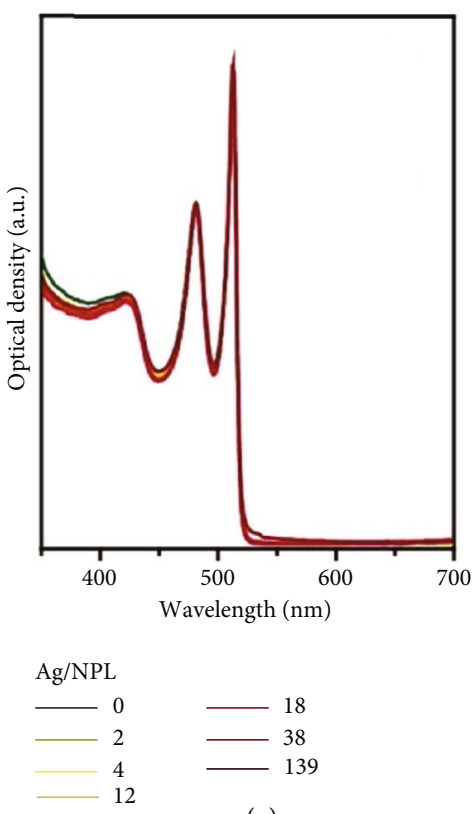

(g)

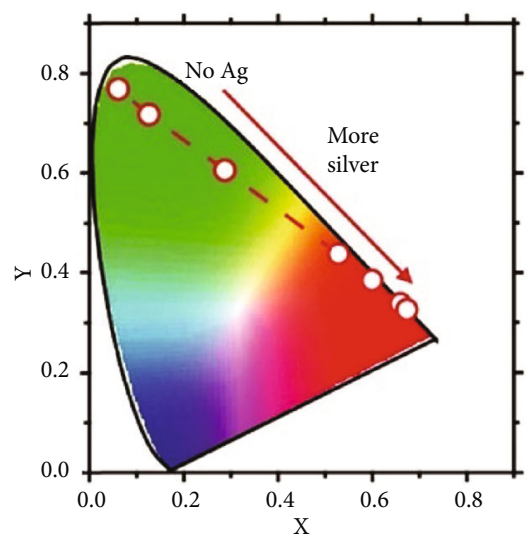

(i)

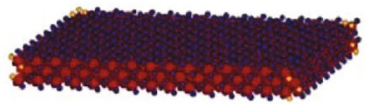

(b)

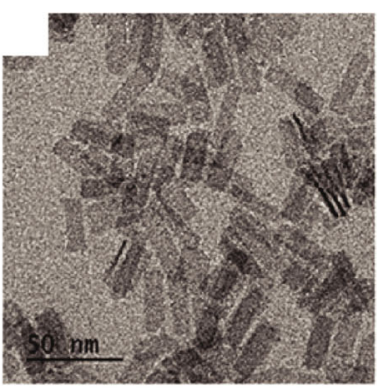

(e)

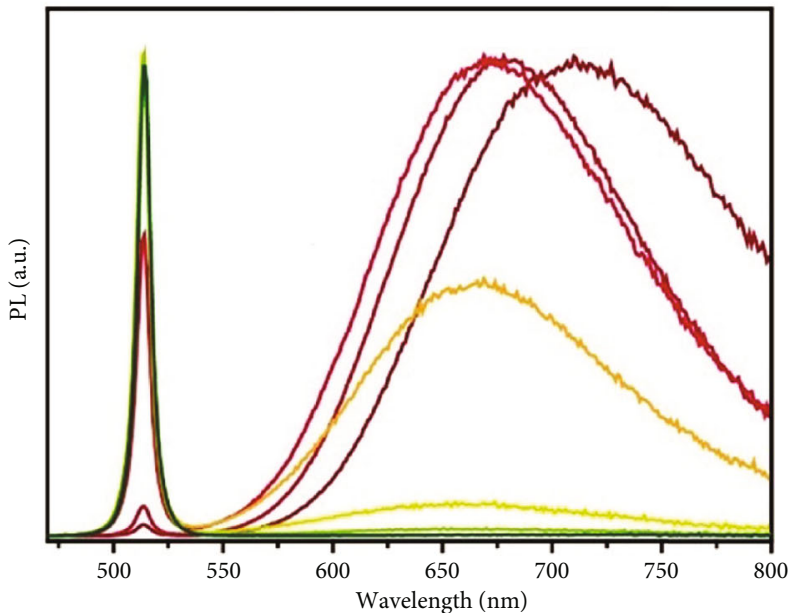

$\begin{array}{rr}\mathrm{Ag} / \mathrm{NPL} & \\ 0 & 18 \\ 2 & -38 \\ 4 & -139 \\ 12 & \end{array}$

(h)

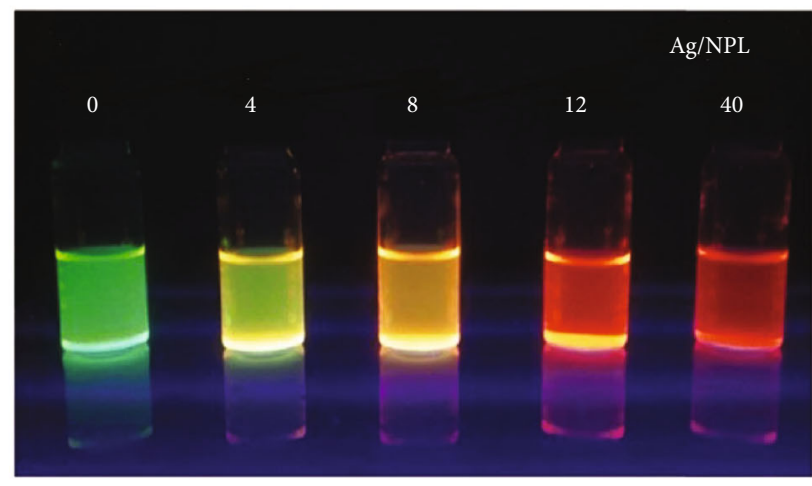

(j)

Figure 4: Scheme of CdSe 2D NPLs (a) without and (b, c) with various amounts of Ag. (d, e, f) TEM of CdSe NPLs with an increasing amount of silver per NPL (0, 18, and 139). (g) UV spectra of CdSe NPLs for various amounts of $\mathrm{Ag}^{+}$doping. (h) Corresponding PL spectra of CdSe NPLs for various amounts of $\mathrm{Ag}^{+}$doped from 0 to $\sim 139$ silver atoms per NPL. (i) Chromaticity diagram highlighting the position associated with the PL spectra of CdSe NPLs with various amounts of $\mathrm{Ag}^{+}$doped. (j) Image of CdSe NPL solution with various amounts of $\mathrm{Ag}^{+}$doped. Adapted with permission from reference [85]. Copyright 2019 American Chemical Society. 


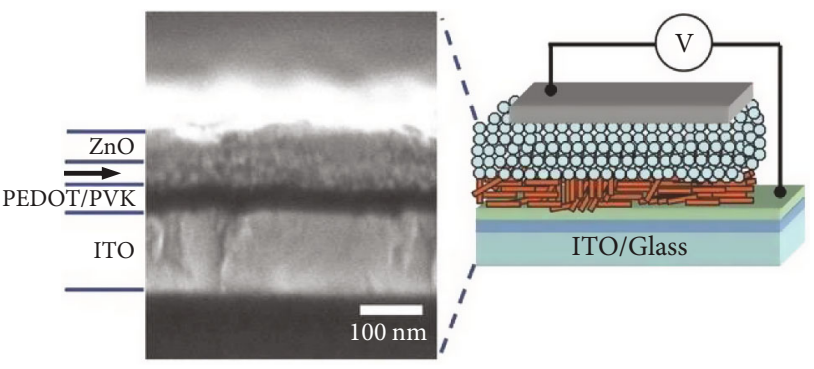

(a)

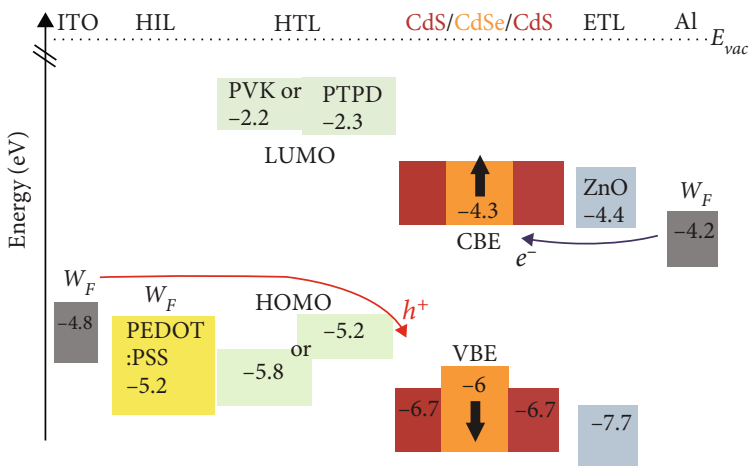

(c)

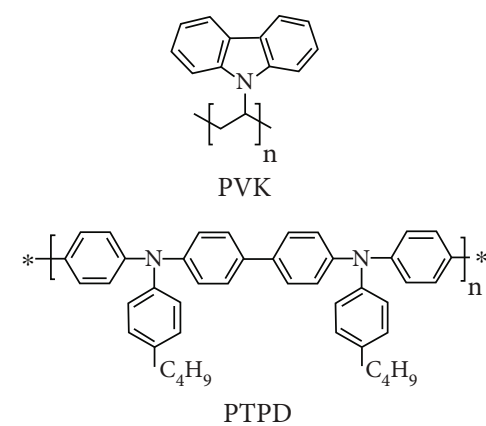

(b)

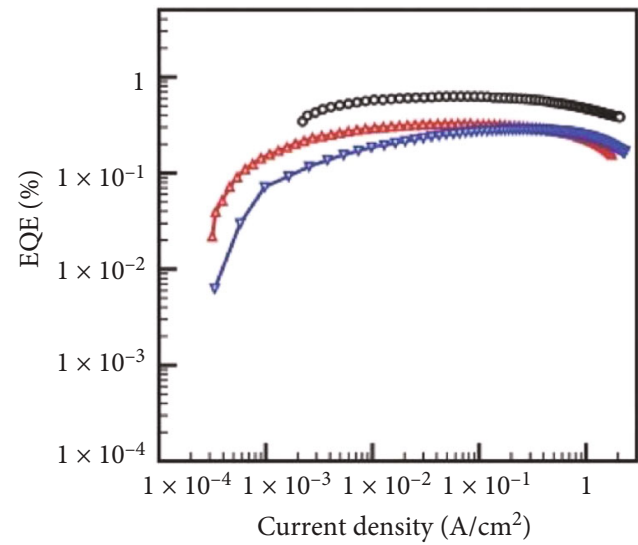

$\begin{array}{ll}\rightarrow & \text { PVK/NPL-MPA/ZnO } \\ \rightarrow & \text { PVK/NPL/ZnO } \\ \rightarrow & \text { PTPD/NPL-MPA/ZnO }\end{array}$

(d)

Figure 5: (a) Right: schematic device architecture of the NPL-LEDs. Left: cross-sectional scanning electron microscopy (SEM) of the hybrid NPL-LED device. (b) The molecular structure for hole transport materials. (c) Energy band diagram of this device's architecture. The black arrow indicates a shift of the band edge because of the quantum confinement. (d) EQE plotted as a function of current density of NPL-LEDs based on different structures. Adapted with permission from reference [4]. Copyright 2013 Wiley-VCH GmbH.

wavelength changed (513 $\mathrm{nm}$ to $481 \mathrm{~nm}$ ) with different $x$ values (0 to 0.67 ). Notably, the PLQY decreased from $37 \%$ to $10 \%$ as more sulfur was alloyed, which was due to the less passivated dangling bonds from the CdS component than those from the CdSe component. Inverted device structure was used with solution-processed $\mathrm{ZnO}$ as ETL and $4,4^{\prime}$-bis(N-carbazolyl)-1,1'-biphenyl (CBP) as HTL. Similar to PL, the EL emission spectrum can also be continuously tuned by varying the Se to $S$ ratio; for example, $496 \mathrm{~nm}, 507 \mathrm{~nm}$, and $520 \mathrm{~nm}$ correspond to $x=0.6,0.34$, and 0 , respectively.

These early demonstrations of NPL-LEDs based on coreonly CdSe NPLs demonstrated the great potential of using NPLs for high efficiency LEDs with narrow emission bandwidth. However, compared with core/shell NPLs, core-only NPLs are less stable and often have lower PLQY. Therefore, the study of NPL-LEDs has been mostly focused on heterostructured core/shell and core/crown NPLs.

4.1.2. Core/Shell CdSe NPL-LEDs. In 2014, Chen et al. reported the first red $(650 \mathrm{~nm})$ LED based on quasi-2D colloidal core/shell CdSe/CdZnS NPLs [4]. In this work, the
CdZnS shell can increase the stability of luminescence properties of CdSe NPLs. The HTL was poly(vinylcarbazole)

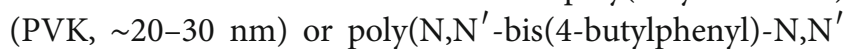
-bis(phenyl)benzidine (PTPD, 30-40 nm) (Figure 5(b)), and the device structure was ITO/PEDOT:PSS/HTL/NPLs $(30-40 \mathrm{~nm}) / \mathrm{ZnO}(60 \mathrm{~nm}) / \mathrm{Al}$ (Figure $5(\mathrm{a}))$. The energy band diagram of the LEDs is shown in Figure 5(c). Two strategies were used to enhance the device performance. To improve the charge injection and transport, the long (i.e., oleic acid) ligands of the as-synthesized NPLs were exchanged to shorter ones (i.e., 3-mercaptopropionic acid), leading to about 2 -fold increase in EQE of $0.63 \%$ and a maximum luminance of $4499 \mathrm{~cd} \mathrm{~m}^{-2}$, although the PLQY decreased by $40 \%$ after the ligand exchange (Figure $5(\mathrm{~d})$ ). Second, the turn-on voltage was reduced from $4.7 \mathrm{~V}$ to $2 \mathrm{~V}$ by increasing the hole mobility of HTL from $\sim 10^{-7}-10^{-6} \mathrm{~cm}^{2} \mathrm{~V}^{-1} \mathrm{~s}^{-1}$ to $\sim 10^{-5} \mathrm{~cm}^{2} \mathrm{~V}^{-1} \mathrm{~s}^{-1}$ through replacing PVK with PTPD (the chemical structures of HTLs are shown in Figure 5(c)). However, this led to a decrease in the maximum EQE $(0.28 \%)$ and luminance $\left(2173 \mathrm{~cd} \mathrm{~m}^{-2}\right)$. A possible reason wasthat the charge balance in PTPD-based NPL-LED was 
poorer than that in PVK-based NPL-LED. Overall, the EL of NPL-based LEDs exhibited very narrow FWHM of 25 to $30 \mathrm{~nm}$, indicating that core/shell NPLs have great potential in achieving pure color for LEDs.

Improved red NPL-LEDs were reported by Giovanella et al. in 2018 [42]. The LEDs used CdSe/CdZnS core/shell NPLs with 4-ML CdSe core and $3 \mathrm{~nm}$ CdZnS shell on each side. These NPLs exhibited PL at $658 \mathrm{~nm}$ and PLQY of $40 \%$. To increase the EQE, a charge-regulating layer of conjugated polyelectrolytes (CPEs) as ETL was introduced to improve the carrier balance as well as the compatibility with the metal cathode. The NPL-LEDs with structure of ITO/ PEDOT:PSS/PVK/NPLs/CPE/Ag emitted red light at $658 \mathrm{~nm}$ with much improved EQE of $5.73 \%$ than that of $0.63 \%$ reported previously [4]. The NPL-LEDs also showed high $1540 \mathrm{~cd} / \mathrm{m}^{2}$ luminance together with FWHM of 25 $\mathrm{nm}$. The CIE color coordinates were measured as (0.71, 0.29 ), corresponding to $98 \%$ saturated red light. All these characteristics demonstrated the potential capabilities of NPL-LEDs for display applications.

In 2019, Kelestemur et al. reported LEDs using CdSe/ $\mathrm{CdS} / \mathrm{CdZnS}$ core/shell NPLs as the emitter [94]. The diagram of band alignment is shown in Figure 6(a) with PVK and $\mathrm{ZnO}$ NPs used as HTL and ETL, respectively. Different from the previous report [4], they used CdSe NPLs with a graded shell consisting of a CdS buffer layer and a $\mathrm{Cd}_{x} \mathrm{Z}$ $\mathrm{n}_{1-x} \mathrm{~S}$ gradient layer (Figure 6(b)). The improved optical properties of the graded shell NPLs showed low amplified spontaneous emission as low as $\sim 40 \mu \mathrm{J} / \mathrm{cm}^{2}$ (Figure 6(c)). The graded shell NPL-LEDs with EL peak at $650 \mathrm{~nm}$ (Figure 6(d)) exhibited much higher EQE (9.92\% vs. $1.80 \%)$ and maximum luminance $\left(46000 \mathrm{~cd} \mathrm{~m}^{-2}\right.$ vs. $10650 \mathrm{~cm} \mathrm{~m}^{-2}$ ) compared with conventional CdSe/CdS NPLs (Figure 6(e)). This was attributed to the imbalanced charge injection caused by the similar conduction band edges of $\mathrm{CdS}$ and $\mathrm{ZnO}$. Thus, the addition of a $\mathrm{Cd}_{x} \mathrm{Zn}_{1-x} \mathrm{~S}$ shell added an additional barrier for electron injection and provided proper surface passivation. The devices exhibited a steep increase in current density and luminance behavior above the turn-on voltage of $\sim 2.3 \mathrm{~V}$.

Recently, Liu et al. reported NPL-LEDs based on CdSe/ CdZnS core/shell NPLs [95]. The device architecture was ITO/ZnO/NPLs/CBP/ $/ \mathrm{MoO}_{3} / \mathrm{Al}$ (Figure $7(\mathrm{a})$ ). Several strategies were used to enhance the device performance. First, the hot-injection shell (HIS) growth of NPLs resulted in a smooth film surface, reduced nonradiative recombination, and enhanced stability. The PLQY was reaching 95\% in solution and $87 \%$ in film even after rigorous cleaning (Figure 7(c)). In comparison, PLQY of core/shell NPLs grown by c-ALD was usually less than $11 \%$, leading to poor device efficiency (Figure 7(d)). Second, the maximum EQE showed strong dependence on the AR where the EQEs of LEDs consisting of NPLs with AR of 3.2, 1.6, and 1.0 (Figure 7(b)) were $5.66 \%, 8.65 \%$, and $11.19 \%$, respectively. This can be attributed to the low root mean square (RMS) roughness of low AR $(2.43 \mathrm{~nm}) \mathrm{NPL}$ films compared to that of high AR $(5.74 \mathrm{~nm})$ and mid-AR $(3.44 \mathrm{~nm})$ NPL films. As a result, the leakage currents were remarkably reduced in low AR NPL-LEDs. Finally, CPB can easily crystallize due to its low glass transition temperature $(\mathrm{Tg})$ of $62^{\circ} \mathrm{C}$, leading to morphological instability of LEDs. To overcome this problem, HTL materials with better stability, $\mathrm{N}, \mathrm{N}^{\prime}$-di-(1-naphthyl)$\mathrm{N}, \mathrm{N}^{\prime}$-diphenyl-(1,1' -biphenyl)-4,4' -diamine (NPB)/4, $4^{\prime}, 4^{\prime \prime}$ -tris(carbazol-9-yl)-triphenylamine (TCTA), were used instead of CPB since TCTA has a $\mathrm{HOMO}(5.7 \mathrm{eV})$ [96] located between the HOMO of NPB $(5.3 \mathrm{eV})$ [97] and the VBM of CdSe/CdZnS. The NPL-LEDs based on HIS NPLs exhibited very high EQE of 19.23\% (Figure 7(e)) and a maximum luminance of $23490 \mathrm{~cd} \mathrm{~m}^{-2}$. This is the first time that the EQE of NPL-LED can be comparable with that of OLEDs and PeLEDs. It is concluded that several conditions need to be satisfied for an ideal NPL-LED. First, the PLQY of NPLs needs to be as high as possible or close to $100 \%$. This can be only achieved by the core/shell structure synthesized by the hotinjection method to passivate nonradiative recombination pathways. Second, the ETL and HTL materials need to be optimized for the best charge injection efficiency and balance. Third, since the major advantage of NPL-LEDs over QLEDs is the in-plane TDM that can break the EQE limit of $20 \%$, successful assembly of NPL films containing only in-plane TDM is critical to realizing the high EQE. We will discuss recent progress towards oriented NPL films in Section 4.3.

Besides red NPL-LEDs studied by most reports, Zhang et al. reported a green LED based on colloidal CdSe/CdS core/shell NPLs with a narrow PL at $556 \mathrm{~nm}$ and FWHM of $12 \mathrm{~nm}$ [98]. They synthesized CdSe core NPLs first and found that the PL spectra shifted to longer wavelengths (from 361 to $556 \mathrm{~nm}$ ) with the increase in reaction time, while the PL FWHM decreased from $30 \mathrm{~nm}$ to $12 \mathrm{~nm}$ at the same time. In order to obtain high-purity green emission, they used the CdSe core with reaction time of $10 \mathrm{~min}$ and PL at $554 \mathrm{~nm}$ to grow CdSe/CdS core/shell NPLs. The growth of shells was along with the edge of CdSe NPLs, which was indicated by the uniform thicknesses of $\sim 2.2 \mathrm{~nm}$ for both core and core/shell NPLs. Therefore, it is possible that these NPLs were core/crown structure instead of core/ shell. The device used a conventional structure of ITO/PEDOT:PSS/TFB/NPL/ZnO/Al (Figure 6(e)). The NPL-LEDs not only exhibited several hundred times increase in brightness but also possessed supernarrow EL with FWHM of $14 \mathrm{~nm}$. The ZnO NP ETL has electron affinity of $\sim 4.3 \mathrm{eV}$ and ionization potential of $\sim 7.6 \mathrm{eV}$, providing both the efficient electron injection channel and barriers confining holes within NPLs (Figure 6(f)). The valence band offset at the NPL/ZnO NP interface led to improved charge recombination efficiency. These devices exhibited maximum luminance efficiency of $12.5 \mathrm{~cd} \mathrm{~A}^{-1}$, corresponding to the highest EQE of $5 \%$ at the brightness of $2000 \mathrm{~cd} \mathrm{~m}^{-2}$. Although the efficiency is lower than those of previous reports which utilized sphere-core/shell QDs, the NPL-based green LEDs still have great potential for green LEDs.

4.1.3. Core/Crown CdSe NPL-LEDs. The heterostructure NPLs can be classified as type I or type II which depends on the band alignment between the core NPLs and the shell or crown [99-100]. In type I NPLs, both electron and hole wave functions are confined in the core as a result of the 


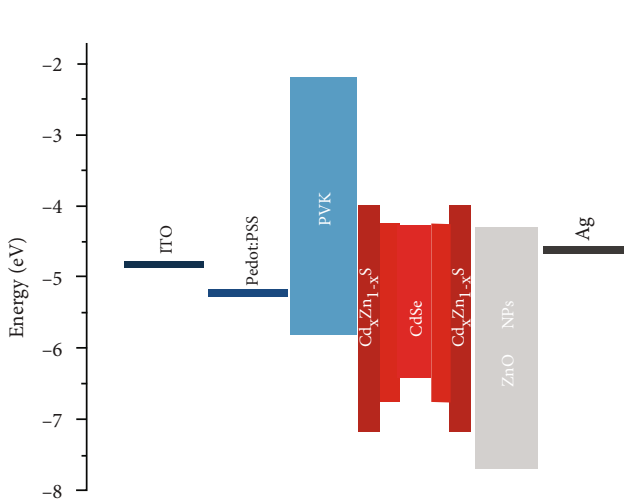

(a)
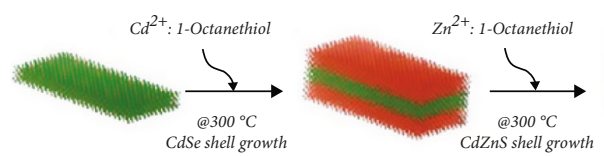

(b)

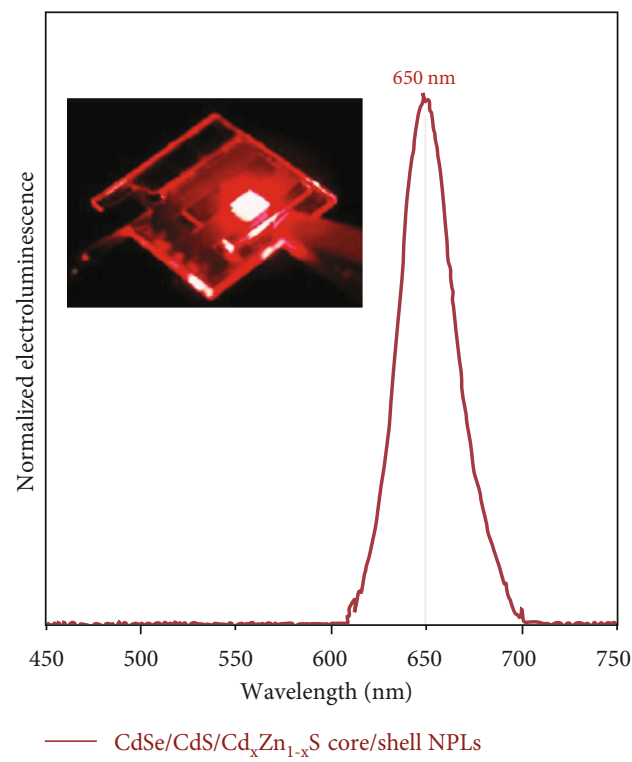

(d)

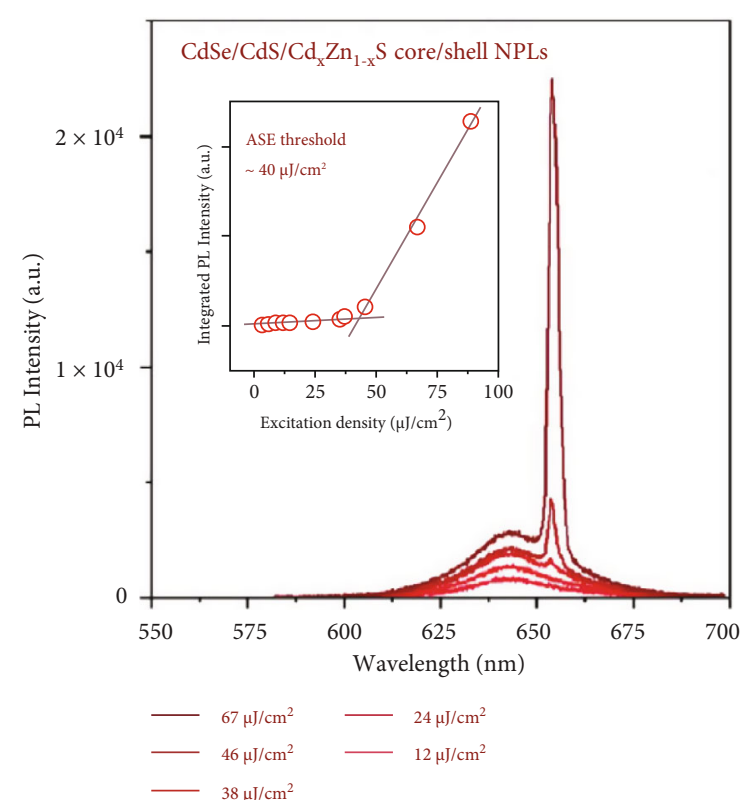

(c)

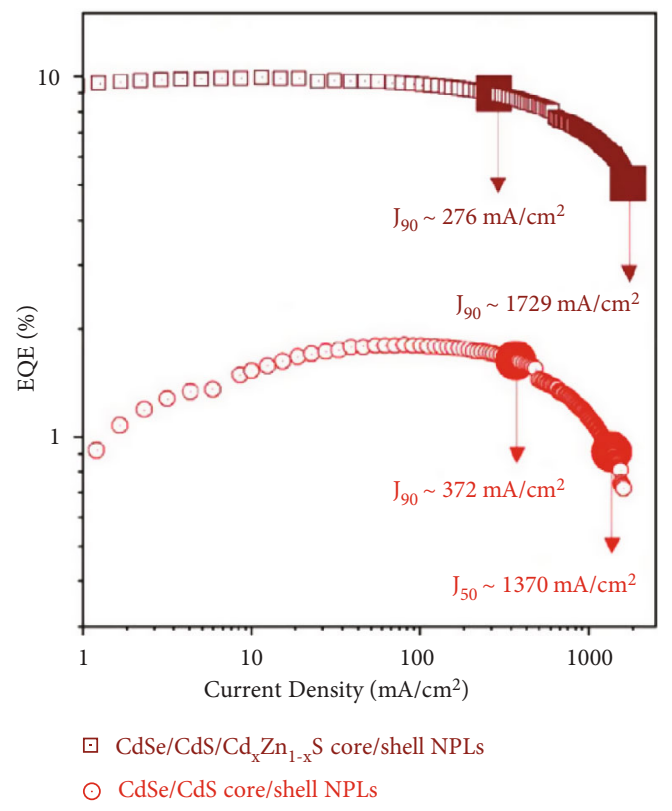

(e)

FIgURE 6: (a) Energy band diagram of the optimized device structure for graded shell CdS/Cd $\mathrm{Zn}_{1-x} \mathrm{~S}$ NPL-LEDs. (b) Schematic of synthesis of graded shell NPLs. (c) Optical gain characteristics of CdSe/CdS/Cd $Z_{x} n_{1-x} S$ NPLs showing a low ASE threshold of $\sim 40 \mu \mathrm{J} / \mathrm{cm}^{2}$. (d) EL spectra of NPL-LEDs. Inset: the photo of the fabricated LED in operation. (e) EQE vs. current density representation of NPL-LEDs. Adapted with permission from reference [94]. Copyright 2019 American Chemical Society

wider band gap of the shell or crown than that of the core. In contrast, the electron and hole wave functions are spatially separated in type II NPLs. Since the first type II NPLs were synthesized in 2015 [101], type II NPLs have shown great potential to develop LEDs with tunable emission colors ranging from the visible to near infrared (NIR). Additionally, type II NPLs can maintain high PLQY and reduced overlap between absorption and emission spectra which is important for achieving high EQE.
In 2018, Liu et al. studied LEDs based on CdSe/ $\mathrm{CdSe}_{0.8} \mathrm{Te}_{0.2}$ core/crown type II NPLs [38]. The PL spectrum of the type II CdSe/CdSe ${ }_{0.8} \mathrm{Te}_{0.2}$ core/crown NPLs showed a red emission peak at $\sim 599 \mathrm{~nm}$. The device used inverted structure of ITO/ZnO/NPLs/TCTA/TPD/MoO$/ \mathrm{Al}$. The high EQE of $3.57 \%$ was ensured by the high PLQY (85\%) of core/crown NPLs. More importantly, the dual HTL of TCTA/TPD worked much better than the conventional CBP or TPD. This is attributed to the following: (i) TCTA 


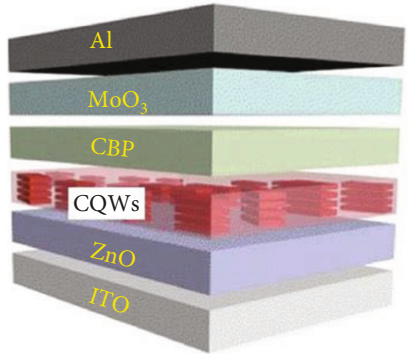

(a)

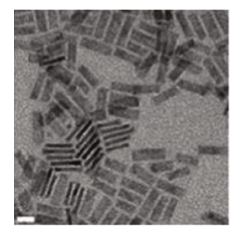

High AR

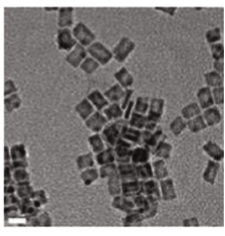

Mid AR

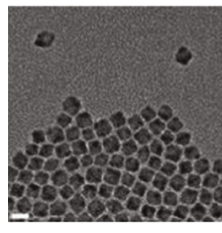

Low AR

(b)

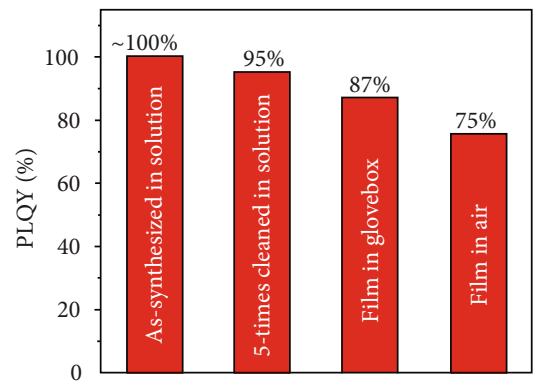

(c)

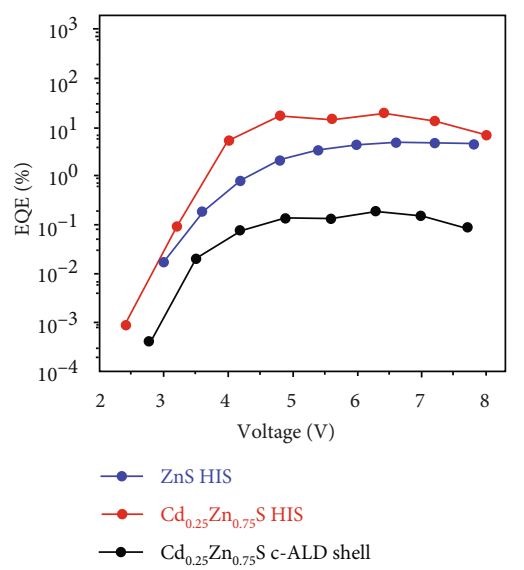

(d)

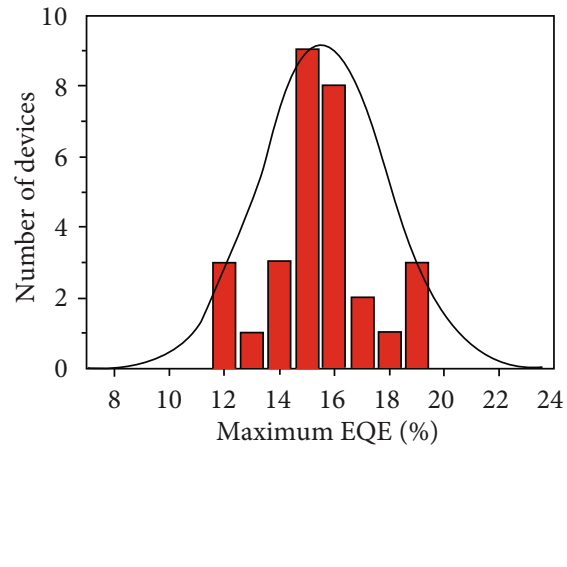

(e)

Figure 7: (a) Device architecture of the NPL-LEDs. (b) HR-TEM images of high, mid, and low AR NPLs. (c) Evolution of the PLQY of core/ shell NPLs. (d) EQE of NPL-LEDs with varying shell composition. (e) EQE histogram of the NPL-LEDs. Adapted with permission from reference [95]. Copyright 2019 Wiley-VCH GmbH.

is hole-dominating and has a higher LUMO to confine the electrons. Hence, more excitons can form when more holes can meet electrons; (ii) the stepwise HOMO of TCTA/TPD reduces the hole barrier and injects more holes into the NPLs; (iii) TCTA/TPD-based NPL-LEDs also have better charge balance, with the high electron injection efficiency owing to the high electron mobility and LUMO barrier of $\mathrm{ZnO}$ [7]; and (iv) the charge neutrality for NPLs can be maintained while keeping good emissive properties due to the dual-hole transport layer [102]. In addition, the inverted structure is more favorable for display applications as the cathode can be directly connected to the low-cost $n$-type metal-oxide or amorphous-silicon thin film transistors [103]. In summary, the core/crown NPL-LEDs exhibited a extremely low turn-on voltage of $1.9 \mathrm{~V}$, a high EQE of $3.57 \%$, and a PE of $9.44 \mathrm{~lm} \mathrm{~W}^{-1}$. Finally, this work demonstrated that it is necessary to focus more on the optimization of device structure.

Recently, Wen et al. reported another core/crown CdSe/ CdS NPL-LED [105]. This device exhibited an electroluminescence (EL) at $521 \mathrm{~nm}$ with a FWHM of $15 \mathrm{~nm}$. The device used inverted architecture of ITO/ZnO/PVP/NPLs/CBP/ $\mathrm{MoO}_{3} / \mathrm{Al}$ due to the better solvent compatibility of $\mathrm{ZnO}$ than HTL materials. The thin PVP layer was introduced to prevent the direct contact between the ETL and the HTL caused by the cracks of the NPL layer. Moreover, the PVP can also slower electron transport and improve the charge balance. As a result, these devices exhibited a maximum luminance efficiency of $4684 \mathrm{~cd} \mathrm{~m}^{-2}$, an EQE of $0.416 \%$, and a current efficiency (CE) of $1.46 \mathrm{~cd} \mathrm{~A}^{-1}$, which were 7fold brightness and 6-fold EQE of those from NPL-LEDs without a PVP layer. This work demonstrated that adding a PVP layer is a great way to enhance performance of NPL-LEDs. The EQE of core/crown NPL-LEDs can be further improved through reducing the stacking NPLs (edgeup orientation). Wen et al. showed that the increasing polar solvent (ethanol) can effectively reduce the staking between NPLs, thus reducing nonradiative energy transfer (NRET) between NPLs (Figure 8(a)) [104]. The NPL-LEDs with structure of ITO/ZnMgO/NPLs/CBP/ $/ \mathrm{MoO}_{3} / \mathrm{Al}$ illuminated pure green light at $521.5 \mathrm{~nm}$ with FWHM of $15 \mathrm{~nm}$, corresponding to CIE coordinates of $(0.108,0.800)$. The suppressed NRET resulted in an EQE of $2.16 \%$ and a maximum luminance of $22500 \mathrm{~cd} \mathrm{~m}^{-2}$, both of which are several times of those from the previous report.

4.1.4. Alloyed Core-Only NPLs for LEDs. One limitation of NPLs is the discrete wavelengths that depend strictly on atomic layer thickness [106-107]. To overcome this problem, Fan et al. used alloyed CdSe NPL with CdS to finely tune the emission spectrum while still leveraging atomicscale thickness control [7]. They used 6-ML CdSe NPLs alloyed with CdS of which the emissions could cover from green to blue (513-481 nm). Different HTL materials, CBP, $\mathrm{NPB}$, and 4,4'-cyclohexylidenebis(N,N-bis (4-methylphenyl)-benzenamine) (TAPC), were compared, and CPB/ 


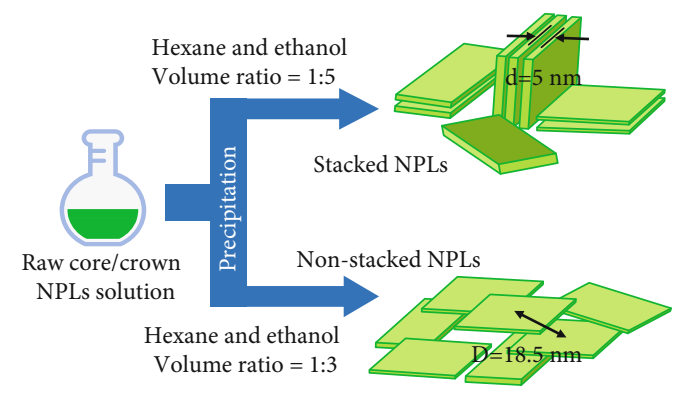

(a)

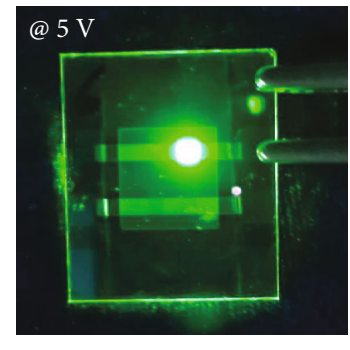

(b)

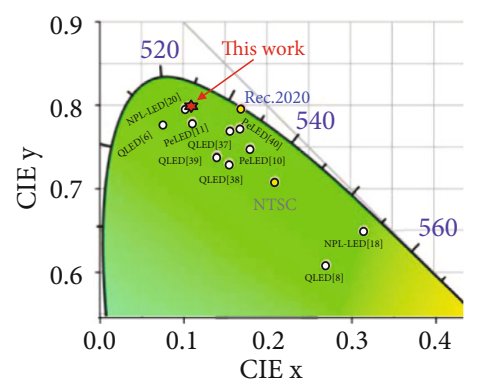

(c)

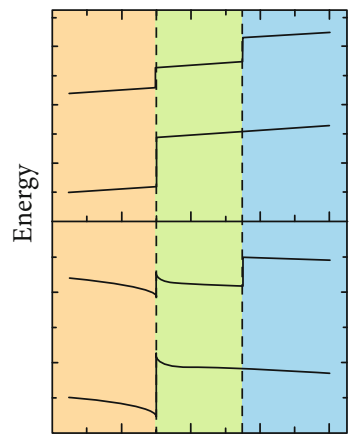

(d)

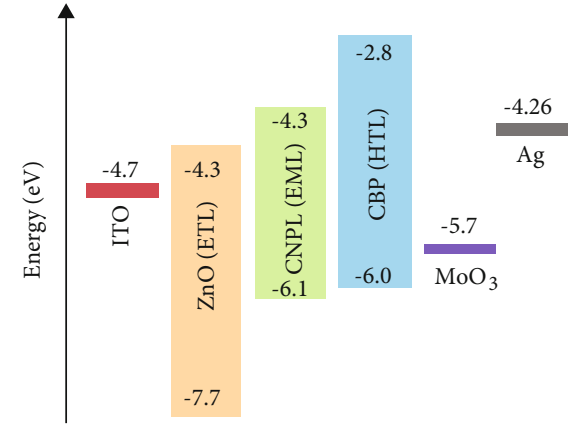

(e)

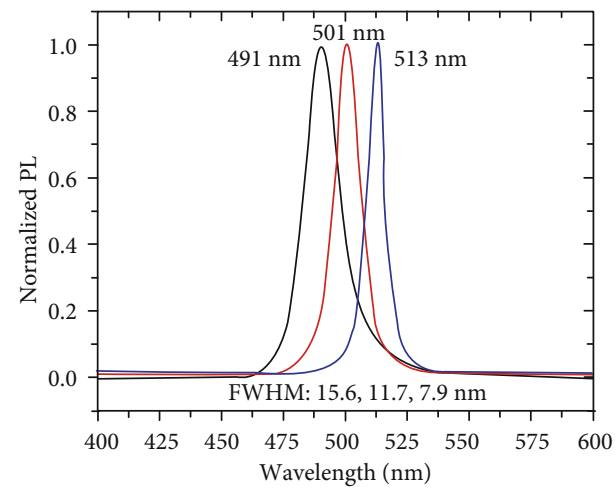

(f)

FIGURE 8: (a) Schematic of reducing the stacking NPLs by solvent optimization. (b) A photograph of NPL-LED emitting pure green light. (c) CIT coordinates of the NPL-LEDs. Adapted with permission from reference [104]. Copyright $2021 \mathrm{Wiley-VCH} \mathrm{GmbH.} \mathrm{(d)} \mathrm{The} \mathrm{CdSe-CdS}$ alloyed NPL-LED under zero bias and forward bias near turn-on voltage (2 V), modeled using SCAPS. (e) Energy band diagram of NPLLEDs. (f) Tunable EL spectra from NPL-LEDs. Adapted with permission from reference [7]. Copyright 2015 American Chemical Society.

$\mathrm{MoO}_{3}$ bilayer HTL was selected for the best performance. The inverted device architecture was ITO/ZnO $(50 \mathrm{~nm}) /$ NPLs $(70 \mathrm{~nm}) / \mathrm{CBP}(65 \mathrm{~nm}) / \mathrm{MoO}_{3}(10 \mathrm{~nm}) / \mathrm{Ag}(100 \mathrm{~nm})$ (Figure $8(\mathrm{~d})$ ). The energy band diagram is shown in Figure $8(\mathrm{e})$. As a result, the NPL-LED showed EL wavelength from $491 \mathrm{~nm}$ to $513 \mathrm{~nm}$ with the narrowest EL FWHM of $12.5 \mathrm{~nm}$ (Figure 8(f)), a peak brightness of $\sim 90 \mathrm{~cd} \mathrm{~m}^{-2}$, and a subband gap turn-on voltage of $2.1 \mathrm{~V}$. This work stated that more efforts were needed to develop the core-only NPLs.

4.2. Perovskite Nanoplatelet-Based LEDs. Metal halide perovskites have attracted a lot of interests for a variety of optoelectronic applications, such as lasers, photovoltaics, and LEDs [108-112]. Particularly, perovskite-based LEDs have drawn lots of attention because of their high PLQY [113-114], narrow emission bandwidth, and tunable EL [115-116]. Since the development of LEDs based on $\mathrm{CH}_{3} \mathrm{NH}_{3} \mathrm{PbBr}_{3}$ perovskite in 2014 [117], many efforts have been made for this new type of LEDs [118-123]. Compared with other materials like organic semiconductors and inorganic quantum dots, perovskites have many advantages, for example, low material cost, easy synthesis, and high color purity.

4.2.1. Hybrid Organic-Inorganic Perovskite NPL-LEDs. Organic-inorganic hybrid perovskites, particularly the threedimensional (3D) perovskites, have emerged as an excellent class of semiconductors due to their high PLQY, long carrier diffusion length, and long carrier lifetime [124-126]. These properties have enabled high-performance LEDs, lasers, solar cells, and many other optoelectronic devices [108, 127-129]. Due to the limited band gap, it has been difficult to achieve blue EL from LEDs based on 3D perovskites. 2D perovskites, on the other hand, have larger band gaps because of the quantum confinement. Liang et al. achieved the first violet LED using $2 \mathrm{D}$ perovskite $\left(\mathrm{C}_{6} \mathrm{H}_{5} \mathrm{CH}_{2} \mathrm{CH}_{2} \mathrm{NH}^{3+}, \mathrm{PEA}\right)_{2} \mathrm{PbBr}_{4}$ thin film [130]. They applied a solvent vapor annealing technique to prepare high-quality micrometer-lateral (PEA) ${ }_{2} \mathrm{PbBr}_{4}$ nanoplatelets. The device structure was ITO/PEDOT:PSS (30nm)/(PEA) ${ }_{2} \mathrm{PbBr}_{4} / 1,3,5$-tris(1-phenyl-1H-benzo[d]imidazol-2-yl) benzene (TPBi, $35 \mathrm{~nm}) / \mathrm{Ca}(25 \mathrm{~nm}) / \mathrm{Al}(100 \mathrm{~nm})$ (Figure 9(a)). The LED displayed an EL emission peak at $410 \mathrm{~nm}$ with FWHM of $14 \mathrm{~nm}$ and an EQE of $0.002 \%$ (Figure 9(b)).

Perovskite NPLs with high crystallinity and high PLQY are critical for NPL-LEDs [130]. Ling et al. synthesized $\mathrm{CH}_{3} \mathrm{NH}_{3} \mathrm{PbBr}_{3}\left(\mathrm{MAPbBr}_{3}\right)$ NPLs with PLQY up to $85 \%$ and fabricated bright perovskite NPL-LEDs [120]. The device structure was ITO/PEDOT:PSS/PVK:2-(4-biphenylyl)-5phenyl-1,3,4-oxadiazole (PBD)/NPLs/bathocuproine (BCP, $50 \mathrm{~nm}) / \mathrm{LiF}(1 \mathrm{~nm}) / \mathrm{Al}(150 \mathrm{~nm})$ (Figure 9(c)). The NPLLEDs exhibited maximum luminance of $10,590 \mathrm{~cd} \mathrm{~m}^{-2}$ $(530 \mathrm{~nm}), \mathrm{PE}$ of $1.0 \mathrm{~lm} \mathrm{~W}^{-1}$, and EQE of $0.48 \%$. Besides high-quality NPLs, bipolar PVK:PBD in the emitting layer 


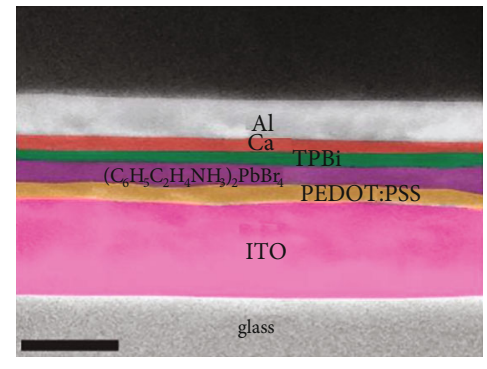

(a)

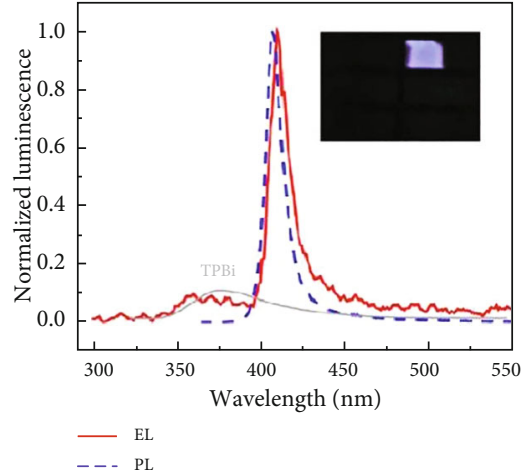

(b)

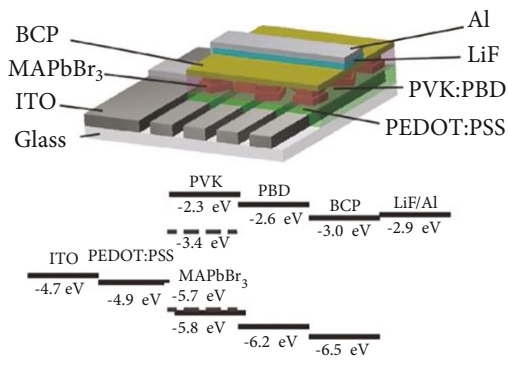

(c)

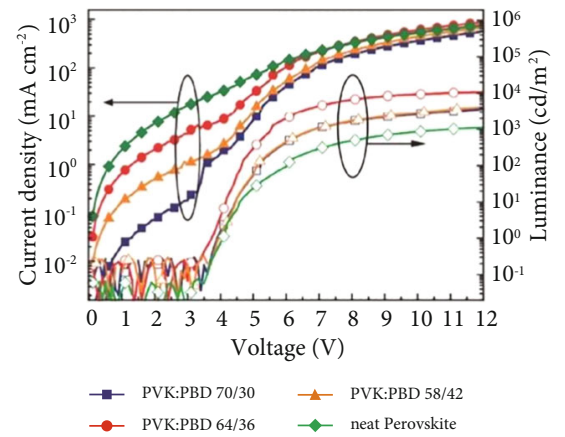

(d)

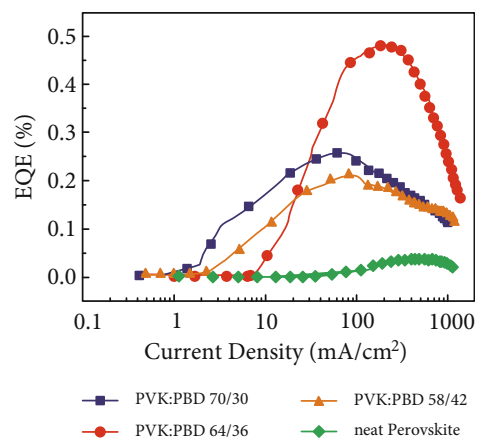

(e)

Figure 9: (a) The SEM for LEDs (scale bar: $200 \mathrm{~nm}$ ). (b) Normalized luminescence of the LED using (PEA) 2 PbBr ${ }_{4}$ NPLs achieved from dimethylformamide vapor annealing at $6 \mathrm{~V}$. EL and PL emission peaks are peaked at 410 and $407 \mathrm{~nm}$, respectively. Inset: a picture for violet light from the (PEA) ${ }_{2} \mathrm{PbB}_{\mathrm{r} 4} \mathrm{LED}$; adapted with permission from reference [130]. Copyright 2016 American Chemical Society. (c) Device architecture and the energy levels of the LED. (d) Current-voltage and luminance-voltage properties of the control LED (neat perovskites without PVK:PBD) and LEDs using the PVK:PBD (ratio of 70:30, 64:36, and 58:42) layer. (e) EQE-current density of LEDs. Adapted with permission from reference [120]. Copyright 2015 Wiley-VCH GmbH.

also improved charge balance (Figures 9(d) and 9(e)). In comparison, the device without the PVK:PBD only showed EQE of $0.038 \%$ and luminance of $1113 \mathrm{~cd} \mathrm{~m}^{-2}$. Additionally, the $\mathrm{MAPbBr}_{3}$ NPLs also showed good stability in air for more than a week.

Similarly, Kumar et al. adopted the $\mathrm{MAPbBr}_{3}$ NPLs as emitters for LEDs [131]. Two strategies were adopted to achieve blue emission from $\mathrm{MAPbBr}_{3}$ NPLs. First, the thickness was precisely controlled by cosurfactant colloidal chemistry to yield a high degree of blue shift. Second, the wide-bandgap and low- $k$ organic hosts (e.g., CBP) as the barrier materials formed perovskite dielectric quantum wells which can enable efficient radiative recombination by the significantly increased exciton binding energy. These results are useful for perovskite LEDs to cover the entire visible spectrum.

Like $\mathrm{MAPbBr}_{3}$, formamidinium lead bromide $\left(\mathrm{FAPbBr}_{3}\right) \mathrm{NPL}$ is also an efficient emitter for LED. Kumar et al. reported a pure green LED using $\mathrm{FAPbBr}_{3}$ NPLs with thickness of 7 10 unit cells [132]. The quantum-confined $\mathrm{FAPbBr}_{3} \mathrm{NPLs}_{\mathrm{N}}$ exhibited high exciton-binding energy of $162 \mathrm{meV}$ through the dielectric quantum well engineering, leading to high PLQY of $92 \%$ in a thin film. By optimizing both the HTLs and ETLs, the best structure of ITO/PEDOT : PSS/poly-TPD/PMMA : FAPbBr ${ }_{3} / 3 \mathrm{TPYMB} / \mathrm{LiF} / \mathrm{Al}$ was selected. As a result, the optimized device showed a maximum current efficiency of $13.02 \mathrm{~cd} \mathrm{~A}^{-1}$ at $529 \mathrm{~nm}$ with a FWHM of $22.8 \mathrm{~nm}$. Moreover, the LED reached CIE 1931 color coordinates of $(0.168,0.773)$ and a wide color gamut covering $97 \%$ and $99 \%$ of the Rec. 2020 standard in the CIE 1931 and the CIE 1976 color space. Compared with the LED without PMMA, the efficiency of the device based on the $2 \mathrm{D} \mathrm{FAPbBr}$-PMMA complex improved by 3 times, which can be attributed to the dielectric confinement effect and the formation of a smoother EML. Moreover, efficient exciton recombination and balanced charge carriers also promoted the device performance due to the following: (i) the deep LUMO of 3TPYMB $(-3.3 \mathrm{eV})$ and the shallow HOMO of poly-TPD $(-5.4 \mathrm{eV})$ can enable the cascade carrier injection; (ii) carrier mobilities in 3TPYMB and poly-TPD are comparable; (iii) the high LUMO of poly-TPD $(-2.0 \mathrm{eV})$ and the low HOMO of 3TPYMB $(-6.8 \mathrm{eV})$ can form an effective carrier confinement.

4.2.2. All-Inorganic Perovskite NPL-LEDs. Hybrid organicinorganic perovskites have been well demonstrated to develop high-efficiency LEDs. However, due to the small formation energy, the organic cations like $\mathrm{CH}_{3} \mathrm{NH}_{3}{ }^{+}$are sensitive to moisture and can easily decompose. On the contrary, 


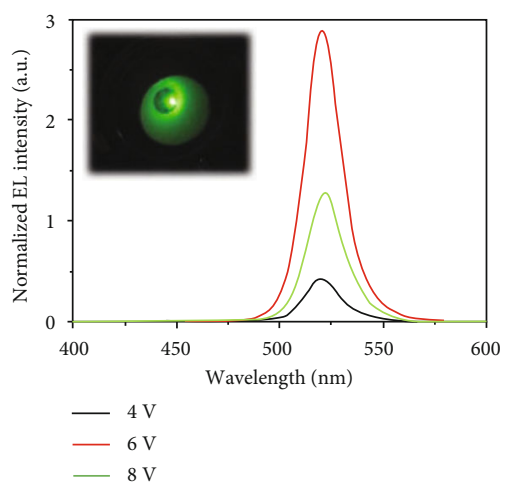

(a)

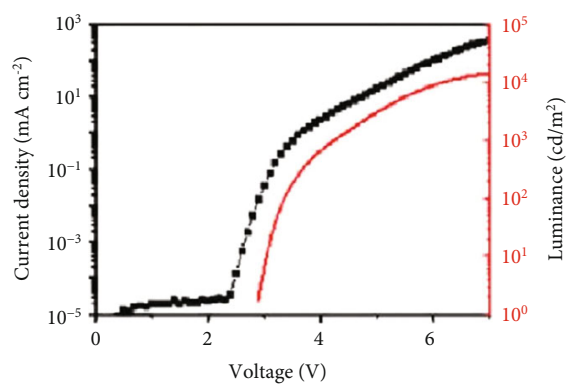

(d)

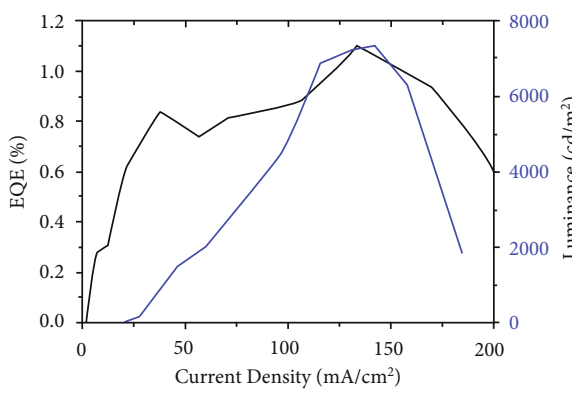

(b)

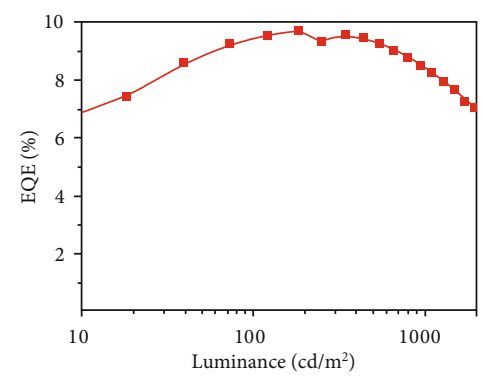

(e)

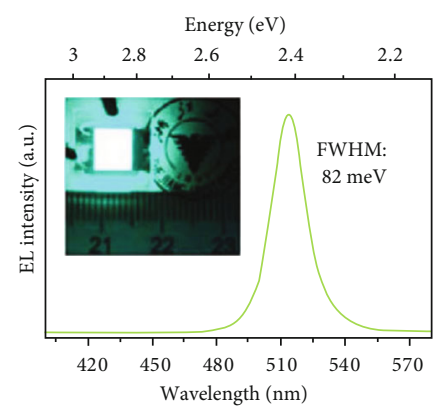

(c)

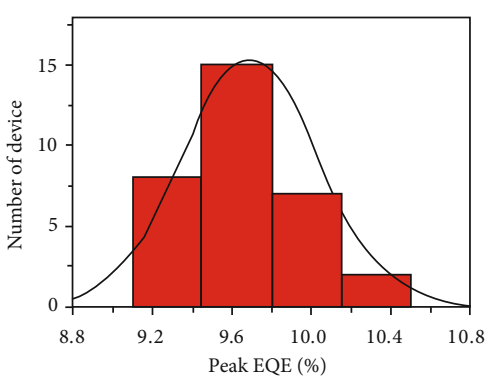

(f)

FIGURE 10: (a) EQE and luminance as a function of current density. (b) EL spectra measured at various voltages. Inset: a picture of LED at $10 \mathrm{~mA} \mathrm{~cm}^{-2}$; adapted with permission from reference [141]. Copyright 2017 American Chemical Society. (c) EL spectrum at an applied bias of $4 \mathrm{~V}$. Inset shows a photograph of a $56 \mathrm{~mm}^{2}$ device. (d) Current density-luminance-voltage characteristics and (e) corresponding EQEluminance curve of a device. (f) Histogram of peak EQEs for 32 devices from five batches. Adapted with permission from reference [143]. Copyright 2019 American Chemical Society.

all-inorganic perovskites like $\mathrm{CsPbBr}_{3}$ may overcome these stability issues while maintaining high PLQY and narrow emission [133-135]. LEDs based on inorganic perovskites have attracted a lot of interest in the past few years [116, 136-139]. Zhang et al. used an inorganic perovskite composite of $\mathrm{CsPbBr}_{3} \mathrm{NCs}$ and quasi-2D CsPb $\mathrm{Br}_{5}$ NPs as emitters and demonstrated LEDs exhibiting a maximum luminance of $3853 \mathrm{~cd} \mathrm{~m}^{-2}$, an EQE of $2.21 \%$, and a current efficiency of $8.98 \mathrm{~cd} \mathrm{~A}^{-1}$, peaking at $527 \mathrm{~nm}$ with a FWHM of $\approx 24 \mathrm{~nm}$ [140]. The device architecture was ITO/PEDOT/CsPbBr ${ }_{3}^{-}$ $\mathrm{CsPb}_{2} \mathrm{Br}_{5} / \mathrm{TPBi} / \mathrm{LiF} / \mathrm{Al}$. Smaller-sized $\mathrm{CsPb}_{2} \mathrm{Br}_{5} \quad \mathrm{NPs}$ attached on the surface of cubic $\mathrm{CsPbr}_{3} \mathrm{NCs}$ can improve current efficiency by reducing exciton diffusion length and enhance emission lifetime by reducing nonradiative energy transfer to trap states. This dual-phase all-inorganic $\mathrm{CsPb}_{2} \mathrm{Br}_{5}-\mathrm{CsPbBr}_{3}$ composite nanocrystal provided a new route of perovskite-based LEDs.

Qin et al. used pure quasi-2D CsPb $\mathrm{Br}_{5}$ NPLs as emitters for LEDs [141]. By centrifugal coating technology, $\mathrm{CsPb}_{2} \mathrm{Br}_{5}$ NPL films with a PLQY of $\sim 35 \%$ were produced. The device architecture was ITO $(100 \mathrm{~nm}) / \mathrm{PEDOT}: \mathrm{PSS}(30 \mathrm{~nm}) /$ $\mathrm{CsPb}_{2} \mathrm{Br}_{5}(30 \mathrm{~nm}) / \mathrm{TPBi}(40 \mathrm{~nm}) / \mathrm{LiF}(0.8 \mathrm{~nm}) / \mathrm{Al}(100 \mathrm{~nm})$. The NPL-LEDs emitted at $520 \mathrm{~nm}$ with a FWHM of $20 \mathrm{~nm}$ (Figure 10(b)) and had a turn-on voltage of $3.8 \mathrm{~V}$, a maximum luminance of $7317 \mathrm{~cd} \mathrm{~m}^{-2}$ (Figure 10(a)), a maximum EQE of $1.1 \%$, and CIE coordinates of $(0.08,0.75)$. Furthermore, $\mathrm{CsPb}_{2} \mathrm{I}_{5}$ NPLs were acquired by halogen exchange, and the $\mathrm{CsPb}_{2} \mathrm{I}_{5}$ LEDs emitted at $685 \mathrm{~nm}$ and had an optical band gap of $1.75 \mathrm{eV}$, a lower PLQY of $\sim 15 \%$, and a maximum EQE of $0.14 \%$ at $20 \mathrm{~mA} \mathrm{~cm}^{-2}$. Similar to this work, Han et al. also studied $\mathrm{CsPb}_{2} \mathrm{Br}_{5}$ NPLs as emitters for LEDs [142]. $\mathrm{CsPb}_{2} \mathrm{Br}_{5}$ NPLs with tunable emission wavelengths from blue $(467 \mathrm{~nm})$ to bright green $(518 \mathrm{~nm})$ were achieved by changing the temperature from $100^{\circ} \mathrm{C}$ to $140^{\circ} \mathrm{C}$. They found that $\mathrm{CsPb}_{2} \mathrm{Br}_{5}$ NPLs synthesized at $140^{\circ} \mathrm{C}$ were capable of emitting narrow-band green light (FWHM $\sim 18 \mathrm{~nm}$ ). Under an operation current of $10 \mathrm{~mA}$, the green LEDs showed a luminous efficiency of $34.49 \mathrm{~lm} / \mathrm{W}$.

$2 \mathrm{D} \mathrm{CsPbX}{ }_{3} \mathrm{NPLs}(\mathrm{X}=\mathrm{I}, \mathrm{Br}$, and $\mathrm{Cl}$ ) have drawn enormous attention since Bekenstein et al. reported $\mathrm{CsPbX}_{3}$ NPLs in 2015 [61]. 2D $\mathrm{CsPbX}_{3}$ NPLs are promising materials for optoelectronic applications due to their precisely tunable thickness [131]. Si et al. reported 2D layered $\mathrm{PBA}_{2}$ $(\mathrm{CsPbBr})_{n-1} \mathrm{PbBr}_{4}$ perovskites $(n=12-16)$ where the $\mathrm{CsPbBr}_{3}$ NPLs were passivated by bulky phenylbutylammonium (PBA) cations [143]. By controlling the $n$ values, the thickness-controlled quantum well (TCQW) $\mathrm{CsPbBr}_{3}$ films were obtained with quantum confinement effects. The device architecture was ITO/NiO/TFB/PVK/CsPbX $3 / \mathrm{Ca} /$ Al. Due to smooth surface features, low trap densities, narrow emission line widths $(\sim 82 \mathrm{meV}$, Figure $10(\mathrm{c}))$, and high PLQY of TCQW CsPbBr ${ }_{3}$ films, the NPL-LEDs exhibited high EQEs of up to $10.4 \%$, maximum luminance of $14000 \mathrm{~cd} \mathrm{~m}^{-2}$ at $8 \mathrm{~V}$, and high color purity (Figures $10(\mathrm{~d})-10(\mathrm{f})$ ). Besides, this 
device also showed good stability because no significant decrease in device efficiency was observed after 30 days in a glovebox. Finally, $\mathrm{CsPbI}_{3}$ NPL-LEDs were obtained by the same approach. The EL spectrum of $\mathrm{CsPbI}_{3}$ NPL-LEDs displayed an emission peak at $683 \mathrm{~nm}$ with a FWHM of $90 \mathrm{meV}$, corresponding to CIE color coordinates of $(0.72,0.28)$ and a peak $\mathrm{EQE}$ of $7.3 \%$.

Tian et al. used quasi-2D $(\mathrm{BA})_{2}(\mathrm{Cs})_{n-1}\left[\mathrm{~Pb}_{n} \mathrm{I}_{3 n+1}\right] /$ poly ( ethylene oxide) (PEO) composite thin films as emitters [144]. The device architecture was ITO/PEDOT:PSS $(40 \mathrm{~nm}) /$ poly-TPD $(20 \mathrm{~nm}) / \mathrm{EL}(50 \mathrm{~nm}) / \mathrm{TPBi}(40 \mathrm{~nm}) / \mathrm{LiF}$ $(1 \mathrm{~nm}) / \mathrm{Al}(100 \mathrm{~nm})$. By changing the molar ratios of organic salt (BAI) to inorganic salts (CsI and $\mathrm{PbI}_{2}$ ), the quasi-2D films with emission at $638-690 \mathrm{~nm}$ were obtained. Due to the surface passivation and small emitting crystals formed in the composite thin films which significantly reduced the nonradiative recombinations, the NPL-LED with emission peak at $680 \mathrm{~nm}$ exhibited a brightness of $1392 \mathrm{~cd} \mathrm{~m}^{-2}$, an EQE of $6.23 \%$, and great stability.

Besides green emitters or red emitters, Yang et al. reported ultrathin $\mathrm{CsPbBr}_{3}$ NPLs for blue LED [145]. The thickness of $\mathrm{CsPbBr}_{3}$ NPLs can be precisely tuned in the monolayer level by controlling the reaction kinetics. Moreover, the edge length can also be varied broadly through modifying the reaction time. The device architecture was ITO/PEDOT: PSS/poly-TPD/CsPbBr 3 /TPBi/LiF/Al. The device showed a maximum luminance of $25 \mathrm{~cd} / \mathrm{m}^{2}$ and an $\mathrm{EQE}$ of $\sim 0.1 \%$. This was the first time that pure $\mathrm{CsPbBr}_{3}$ NPLs were used as the blue LED, and more efforts are still required to further improve the performance.

Hoye et al. demonstrated that the key limiting factors of blue-emitting NPL perovskite LEDs were the large barriers for hole injection and substantial nonradiative decay at the NPL/HTL interface [146]. Both issues arose in part due to the deep ionization potentials $(\geq 6.5 \mathrm{eV})$ of NPLs, leading to the more difficult hole injection than electron injection and resulting in higher electron current density than hole current density. To overcome these issues, they used a poly(triarylamine) interlayer (poly-TPD for sky blue NPLs; TFB for blue NPLs) between the PEDOT: PSS layer and emitter layer to reduce the nonradiative loss and increase hole injection efficiency. As a result, the EQE of the blue $(464 \mathrm{~nm})$ and sky blue $(489 \mathrm{~nm})$ LEDs were increased to $0.3 \%$ and $0.55 \%$ which were higher than previous reports.

For further development, Zhang et al. used the surface ligand engineering strategy to enhance the PLQY and stability of perovskite NPLs [147]. The device architecture was ITO/PEDOT: $\mathrm{PSS} /($ poly-TPD)/CsPbBr $/ 3$ /TPBi/LiF/Al

(Figure 11(a)). By replacing the original long-chain OLA with halide ion-pair ligand (didodecyl dimethyl ammonium bromide (DDAB)), significant enhancement of PLQY and stability was achieved while no significant change was observed in morphology and crystal structure of the NPLs. Due to the halide ion-pair ligand, the $\mathrm{CsPbBr}_{3} \mathrm{NPL}$ films were more uniform with a lower RMS roughness $(0.99 \mathrm{~nm})$ compared with NPLs with OLA ligands $(1.60 \mathrm{~nm})$. Besides, the hole injection barrier between the poly-TPD and the $\mathrm{CsPbBr}{ }_{3}$ NPLs was reduced. After the ligand exchange, the $\mathrm{CsPbBr}_{3}$ NPL-LEDs showed maximum luminance of
$41.8 \mathrm{~cd} / \mathrm{m}^{2}$, peak EQE of $0.56 \%$, and PE of $0.4 \mathrm{~lm} / \mathrm{W}$. Since the remaining hole injection barrier $(1.5 \mathrm{eV})$ was still relatively large, they inserted a thin CBP layer as another HTL between the $\mathrm{CsPbBr}_{3}$ NPLs and the poly-TPD HTL to further improve the charge balance and facilitate the hole injection. Consequently, the peak EQE reached $1.42 \%$ and $\mathrm{PE}$ reached $1.33 \mathrm{~lm} / \mathrm{W}$ (Figures 11(c)-11(f)). This work demonstrated that the surface ligand engineering played an important role in improving PLQY and stability of perovskite NPLs and the performance of LEDs.

4.3. Oriented NPL Films for Improved Out-Coupling Efficiency. NPLs are considered promising materials for LEDs mainly because the in-plane TDMs provide new opportunities to increase the out-coupling efficiency and thus break the EQE limit of $20 \%$ for QLEDs [148]. Compared with isotropic NCs (QDs) which are difficult to align their TMDs due to the isotropic shape, the anisotropic NPLs can be assembled into uniform film with unity in-plane TDM distribution; that is, all NPLs are in face-down orientation. Recently, several reports have demonstrated controlled assembly of NPLs with all TDMs aligned in-plane [149-151]. Additionally, NPL-LEDs with oriented NPL films were also proven with EQEs up to 24.2\% [152-153]. Gao et al. used liquid-liquid interfacial assembly to control the orientation of CdSe NPLs [149]. The monolayer CdSe NPL film can be assembled by adding the NPLs in hexane dispersion to the surface of diethylene glycol (DEG). Then, the monolayer film can be transferred to a substrate submerged in DEG as the DEG was slowly drained from the bottom of the container. It was found that the amount of additional oleic acid added to the DEG was critical to determining the orientation of NPLs by tuning the interfacial energy between the NPLs and the NPL-DEG interface. However, the insulating additive may affect the charge transport. To overcome this issue, Momper et al. developed an improved method through controlled solvent evaporation [150]. They select acetonitrile instead of DEG for the higher interaction potential between the NPL flat surface and the liquid. In addition, the evaporation rate of the solvent where NPLs were dispersed in was also critical, where fast evaporation either by increasing the temperature or selecting high vapor pressure solvent can result in face-down assembly.

In case of perovskite NPLs, Kumar et al. reported efficient NPL-LEDs based on oriented $\mathrm{FA}_{0.5} \mathrm{MA}_{0.5} \mathrm{PbBr}_{3}$ NPLs [152]. The perovskite NPLs were directly assembled on the substrate by spin coating. Owing to the shear-induced ordering during spin coating, the majority of NPLs were in facedown orientation. Comparably, drop casting resulted in random orientation containing both face-down and edge-up orientations. The NPL-LEDs with optimized device structure of ITO/PEDOT: PSS/X-F6-TAPC/NPLs/3TPYMB/LiF/ $\mathrm{Al}$ exhibited record high EQE of $24.2 \%$ covering a wide range of luminance between 30 and $1500 \mathrm{~cd}^{-2}$. The emission wavelength was at $528 \mathrm{~nm}$ with a FWHM of $22 \mathrm{~nm}$. Recently, Cui et al. also reported perovskite NPL-LEDs using oriented inorganic perovskite $\mathrm{CsPbBr}{ }_{3} \mathrm{NPLs}$ [153]. The $\mathrm{CsPbBr}_{3}$ NPLs were directly grown on the PVK HTL by spin coating the precursor solution followed by annealing at 


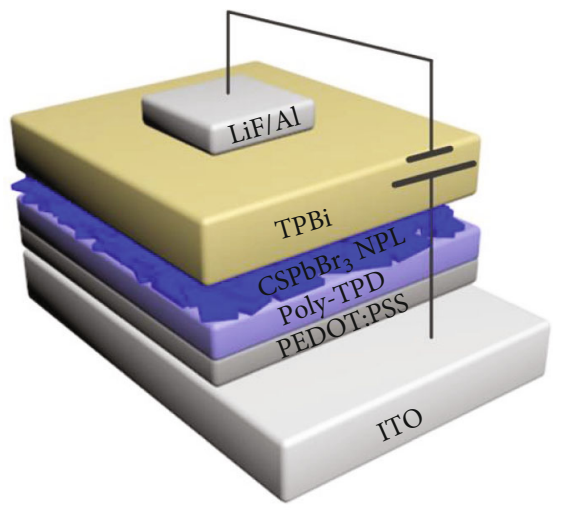

(a)

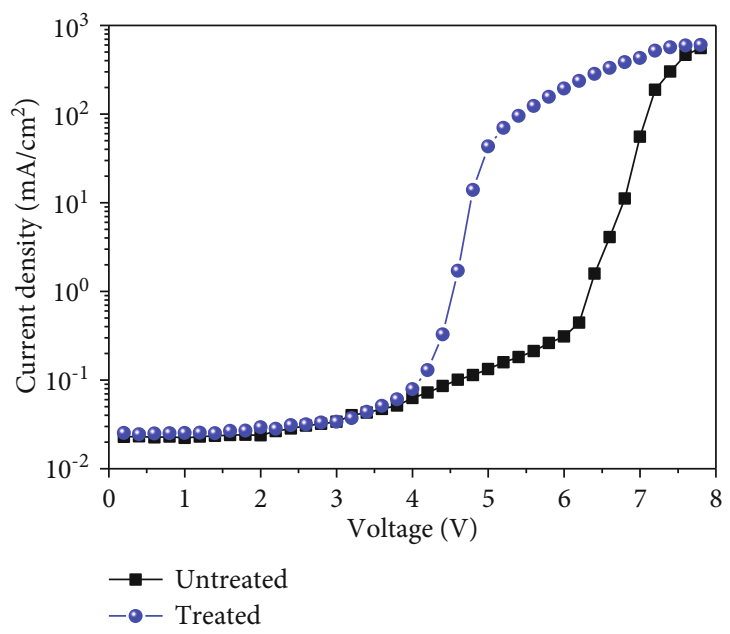

(c)

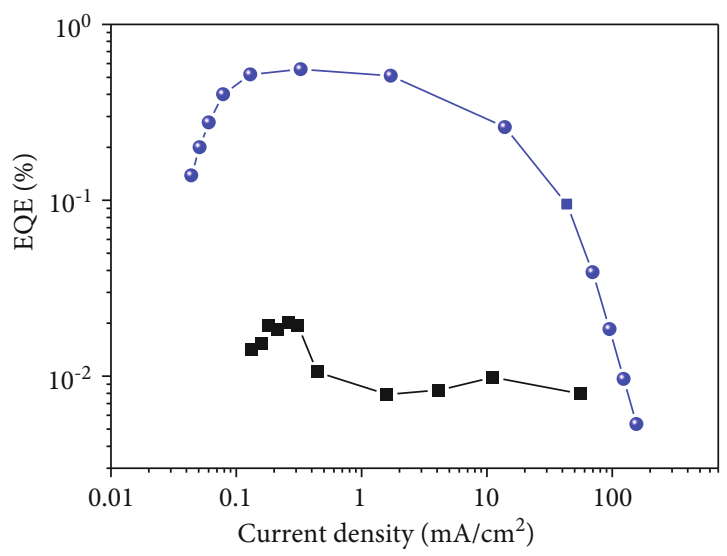

(e)

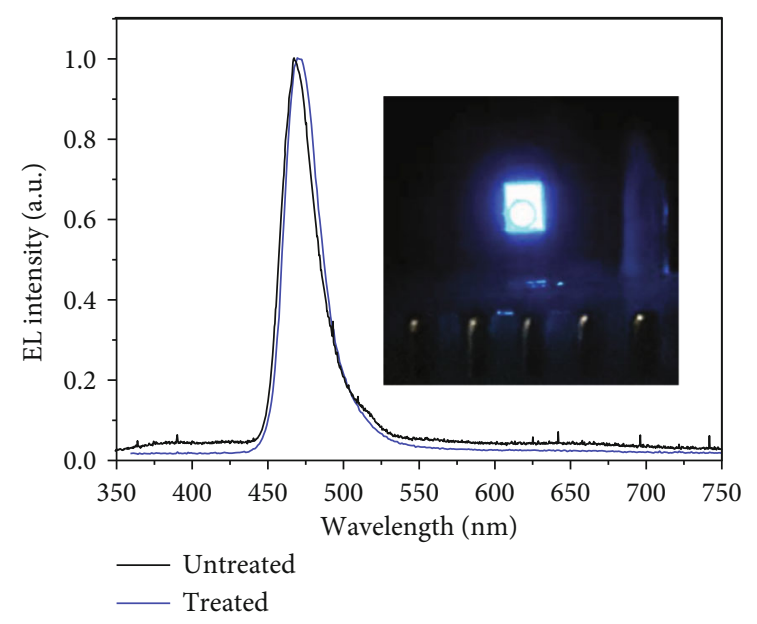

(b)

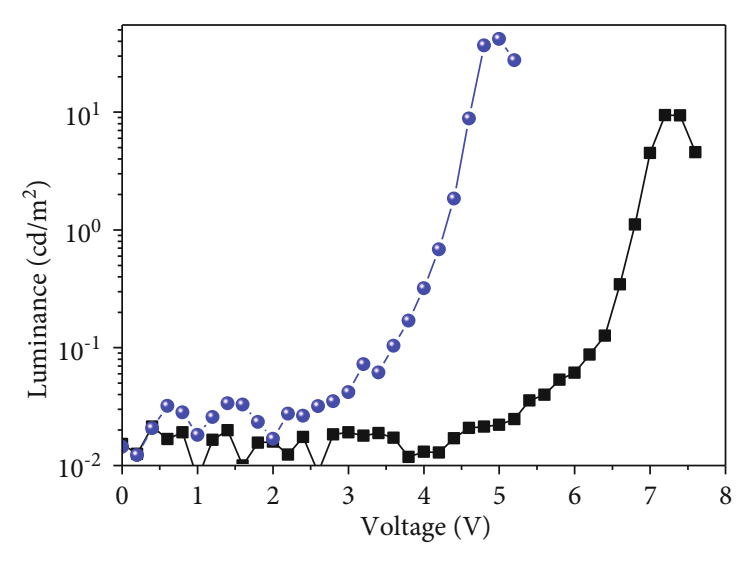

(d)

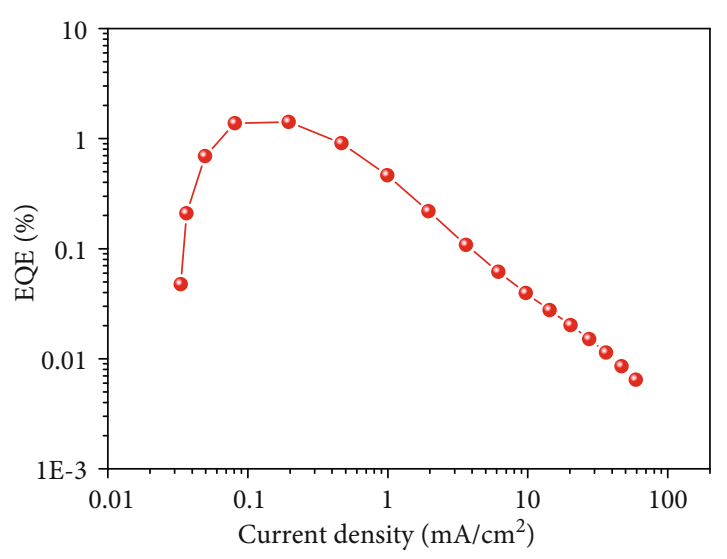

(f)

Figure 11: (a) Schematic device architecture of the $\mathrm{CsPbBr}_{3}$ NPL-based LED. (b) EL spectra of the untreated and treated CsPbBr $\mathrm{NPL}_{3}$ based LEDs. Inset: a photograph of a working treated $\mathrm{CsPbr}_{3}$ NPL-based LED at a driving voltage of $5 \mathrm{~V}$. (c) Current density-voltage, (d) luminance-voltage, and (e) external quantum efficiency-current density curves of the untreated and treated $\mathrm{CsPbBr} \mathrm{NPL}_{3} \mathrm{Nased}$ LEDs. (f) External quantum efficiency-current density curve of the treated $\mathrm{CsPbBr}_{3}$ NPL-based LED with a bilayer HTL. Adapted with permission from reference [147]. Copyright 2019 American Chemical Society. 
TABLE 2: Summary of the performance of representative NPL-LEDs.

\begin{tabular}{|c|c|c|c|c|c|}
\hline Chemical composition & $\lambda(\mathrm{nm})$ & PLQY (\%) & EQE (\%) & CIE & $\overline{\text { Ref }}$ \\
\hline CdSe & 515 & 30 & 0.63 & - & 4 \\
\hline CdSe/CdS/CdZnS (core/shell) & 646 & 89 & 9.92 & $(0.73,0.29)$ & 94 \\
\hline CdSe/CdZnS (core/shell) & 650 & 95 & 19.23 & $(0.715,0.283)$ & 95 \\
\hline CdSe/CdS (core/shell) & 556 & 85 & 5 & $(0.316,0.651)$ & 98 \\
\hline $\mathrm{CdSe} / \mathrm{CdSe}_{0.8} \mathrm{Te}_{0.2}$ (core/crown) & 599 & 85 & 3.57 & $(0.61,0.38)$ & 38 \\
\hline CdSe/CdS (core/crown) & 521 & 84 & 0.416 & $(0.101,0.806)$ & 105 \\
\hline CdSe-CdS (alloyed) & $481-513$ & - & - & - & 7 \\
\hline$(\mathrm{PEA})_{2} \mathrm{PbBr}_{4} \mathrm{NPL}$ & 410 & 85 & 0.002 & - & 130 \\
\hline $\mathrm{MAPbBr}_{3}$ & 530 & 85 & 0.48 & - & 120 \\
\hline $\mathrm{FAPbBr}_{3}$ & 529 & 92 & 2.21 & $(0.168,0.773)$ & 132 \\
\hline 2D CsPb ${ }_{2} \mathrm{Br}_{5}-3 \mathrm{D} \mathrm{CsPbBr}{ }_{3}$ & 527 & 83 & 2.21 & - & 140 \\
\hline $\mathrm{CsPb}_{2} \mathrm{Br}_{5}$ & 520 & 35 & 1.1 & $(0.08,0.75)$ & 141 \\
\hline $\mathrm{CsPb}_{2} \mathrm{Br}_{5}$ & $467-518$ & 63 & - & $(0.25,0.69)$ & 142 \\
\hline $\mathrm{PBA}_{2}\left(\mathrm{CsPbBr}_{3}\right)_{n-1} \mathrm{PbBr}_{4}$ & 514 & 55 & 9.7 & $(0.07,0.74)$ & 143 \\
\hline $\mathrm{CsPbBr}_{3}$ & 480 & - & 0.1 & - & 145 \\
\hline $\mathrm{CsPbBr}_{3}$ & 464 & 19 & 0.3 & - & 146 \\
\hline $\mathrm{CsPbBr}_{3}$ & 489 & 27 & 0.55 & - & 146 \\
\hline $\mathrm{CsPbBr}_{3}$ & 469 & 69 & 1.42 & & 147 \\
\hline $\mathrm{FA}_{0.5} \mathrm{MA}_{0.5} \mathrm{PbBr}_{3}$ & 528 & - & 24.2 & - & 152 \\
\hline $\mathrm{CsPbBr}_{3}$ & 518 & 75 & 23.6 & $(0.09,0.78)$ & 153 \\
\hline
\end{tabular}

$100^{\circ} \mathrm{C}$. The key factor affecting the growth of face-down NPLs was found to be the bulky organic ammonium cations in the precursor solution which was prepared by mixing $\mathrm{PBABr}, \mathrm{PEABr}, \mathrm{CsBr}$, and $\mathrm{PbBr}_{2}$ in $\mathrm{DMSO}$. Although not completely understood yet, the formation of face-down NPLs may originate from the van der Waals interactions. It was also found that adding $\mathrm{LiBr}$ into the precursor solution can significantly improve the PLQY from $\sim 50 \%$ to $75 \%$ due to the better surface passivation. The NPL-LEDs adopted the device structure of $\mathrm{ITO} / \mathrm{NiO}_{x} / \mathrm{TFB} / \mathrm{PVK} /$ NPLs/TPBi/LiF/Al which demonstrated a high EQE of $23.6 \%$ from a champion device and an average EQE of $21.3 \%$ from 36 devices. The emission was at $518 \mathrm{~nm}$ with a narrow FWHM of $16 \mathrm{~nm}$, corresponding to CIE color coordinates of $(0.09,0.78)$. With further development, the EQE of oriented NPL-LEDs is expected to reach the upper limit of $40 \%$ very soon.

\section{Summary and Outlook}

Over the past few years, NPL-LED technology has experienced fast development, especially in the synthesis of NPLs. Due to their unique optoelectronic properties, NPLs have attracted significant attention for various fields, and many efforts have been made to improve the properties including PLQY, FWHM, and stability. For NPL-LEDs, the charge carrier balance is a critical factor affecting the performance. Through modulating potential barriers, charge balance in EMLs can be improved, resulting in high formation probability of excitons, low nonradiative recombination rates, and reduced overflow current of excess carriers. Optimizing the structure or composition of NPLs, for example, core/ shell, core/crown, and alloyed NPLs, can increase PLQY by prohibiting nonradiative recombination. For NPL-LEDs, understanding the interfacial physics is critical to enhance the performance. The exciton quenching at the interface of charge transport layer/NPLs has been greatly suppressed, and the potential energy barrier has been reduced via modifying the interface of NCs films or HTL (e.g., ligand exchange). In virtue of these advances, the EQEs of red NPL-LEDs based on CdSe/ZnCdS NPLs have reached nearly $20 \%$. Although the performance of NPL-LEDs has been significantly enhanced, they still lag behind state-of-the-art spherical QD-LEDs and OLEDs. However, it is believed that NPL-LEDs have the potential to be comparable to their counterparts. Table 2 summarizes the properties and performance of different NPL-LEDs. CdSe-based NPLs have been shown as great EL for red and green LEDs. Due to the intrinsically low band gap of CdSe, it is challenging to achieve blue or deep blue LED from CdSe NPLs. On the other hand, perovskite NPLs, consisting of a large family of perovskite materials, can potentially cover the entire color space required for LEDs. Currently, the research of perovskite NPL-LEDs has been focusing on inorganic $\mathrm{CsPbBr}_{3}$ due to the simpler synthesis and better stability compared with its organic-inorganic counterparts. However, the perovskite NPL-LEDs still suffer very limited lifetime compared with CdSe NPL-LEDs. Nevertheless, the study of NPL-LEDs only emerged less than a decade ago; thus, many fundamental issues remain unsolved and urge further exploration.

There are a few issues still waiting to be solved before NPLLEDs can be successfully commercialized. First, although CdSe- 
based NPL-LEDs have exhibited higher EQE for red and green emission, the toxicity of $\mathrm{Cd}$ strictly limited the use of $\mathrm{Cd}$ containing NCs in practical applications. Thus, the studies of environmentally friendly non-Cd-containing NCs and their LEDs are urgently required. Second, many improvements are left to be done for NPL-LEDs, such as the EQE, PE, and lifetime. These can be achieved by employing NPLs with high PLQY and stability, carefully manipulating charge distribution, and introducing the out-coupling technique [154-155]. In addition, energy barrier and material selection also need to be managed [156-158]. Finally, blue NPL-LEDs currently lag behind in device efficiency and lifetime compared to green and red NPL-LEDs. The longest operation lifetimes of blue NPL-LEDs are far below the minimum requirement for display applications $(10000 \mathrm{~h})$, primarily due to the low-quality blue emitting materials with deep-lying VBM, which is not sufficient for efficient hole flow across the device. Hence, new HTL materials with deeper HOMO/VBM level as well as higher mobility are required to realize charge balance and enhance the lifetime and the efficiency of blue NPL-LEDs. Moreover, surface modification can be introduced to reduce the potential energy barrier and improve the performance of NPL-LEDs.

Despite many challenges remaining unsolved at present, with further research and development of the NPL-LEDs, anisotropic nanocrystals are promising materials to be widely applied in display- and light-emitting-related applications in the future.

\section{Conflicts of Interest}

The authors declare no conflict of interest regarding the publication of this article.

\section{Authors' Contributions}

Yating Guo and Feng Gao contributed equally to this work

\section{Acknowledgments}

This work is supported by the National Natural Science Foundation of China $(22179009,22105018,22005034$, and 21811530054) and Beijing Institute of Technology.

\section{References}

[1] Y. Shirasaki, G. J. Supran, M. G. Bawendi, and V. Bulović, "Emergence of colloidal quantum-dot light-emitting technologies," Nature Photonics, vol. 7, no. 1, pp. 13-23, 2013.

[2] Y. Chen, J. Vela, H. Htoon et al., "“Giant” multishell CdSe nanocrystal quantum dots with suppressed blinking," Journal of the American Chemical Society, vol. 130, no. 15, pp. 50265027, 2008.

[3] X. Dai, Z. Zhang, Y. Jin et al., "Solution-processed, highperformance light-emitting diodes based on quantum dots," Nature, vol. 515, no. 7525, pp. 96-99, 2014.

[4] Z. Chen, B. Nadal, B. Mahler, H. Aubin, and B. Dubertret, "Quasi-2D colloidal semiconductor nanoplatelets for narrow electroluminescence," Advanced Functional Materials, vol. 24, no. 3, pp. 295-302, 2014.
[5] B. Guzelturk, O. Erdem, M. Olutas, Y. Kelestemur, and H. V. Demir, "Stacking in colloidal nanoplatelets: tuning excitonic properties," ACS Nano, vol. 8, no. 12, pp. 12524-12533, 2014.

[6] C. She, I. Fedin, D. S. Dolzhnikov et al., "Red, yellow, green, and blue amplified spontaneous emission and lasing using colloidal CdSe nanoplatelets," ACS Nano, vol. 9, no. 10, pp. 9475-9485, 2015.

[7] F. Fan, P. Kanjanaboos, M. Saravanapavanantham et al., "Colloidal CdSe1-xSx Nanoplatelets with narrow and continuously-tunable electroluminescence," Nano Letters, vol. 15, no. 7, pp. 4611-4615, 2015.

[8] V. L. Colvin, M. C. Schlamp, and A. P. Alivisatos, "Lightemitting diodes made from cadmium selenide nanocrystals and a semiconducting polymer," Nature, vol. 370 , no. 6488 , pp. 354-357, 1994.

[9] T.-H. Kim, K.-S. Cho, E. K. Lee et al., "Full-colour quantum dot displays fabricated by transfer printing," Nature Photonics, vol. 5, no. 3, pp. 176-182, 2011.

[10] W. K. Bae, Y.-S. Park, J. Lim et al., "Controlling the influence of Auger recombination on the performance of quantum-dot light-emitting diodes," Nature Communications, vol. 4, no. 1, p. 2661, 2013.

[11] S. Delikanli, G. Yu, A. Yeltik et al., "Ultrathin highly luminescent two-monolayer colloidal CdSe nanoplatelets," Advanced Functional Materials, vol. 29, no. 35, article 1901028, 2019.

[12] K.-H. Lee, C.-Y. Han, H.-D. Kang et al., "Highly efficient, color-reproducible full-color electroluminescent devices based on red/green/blue quantum dot-mixed multilayer," ACS Nano, vol. 9, no. 11, pp. 10941-10949, 2015.

[13] E. Jang, S. Jun, H. Jang, J. Lim, B. Kim, and Y. Kim, "Whitelight-emitting diodes with quantum dot color converters for display backlights," Advanced Materials, vol. 22, no. 28, pp. 3076-3080, 2010.

[14] J. M. Pietryga, Y.-S. Park, J. Lim et al., "Spectroscopic and device aspects of nanocrystal quantum dots," Chemical Reviews, vol. 116, no. 18, pp. 10513-10622, 2016.

[15] J. Frischeisen, D. Yokoyama, A. Endo, C. Adachi, and W. Brütting, "Increased light outcoupling Efficiency in dyedoped small molecule organic light-emitting diodes with horizontally oriented emitters," Organic Electronics, vol. 12, no. 5, pp. 809-817, 2011.

[16] K.-H. Kim and J.-J. Kim, "Origin and control of orientation of phosphorescent and TADF dyes for highefficiency OLEDs," Advanced Materials, vol. 30, no. 42, article 1705600, 2018.

[17] H. Greiner, "Light extraction from organic light emitting diode substrates: simulation and experiment," Japanese Journal of Applied Physics, vol. 46, no. 7A, pp. 4125-4137, 2007.

[18] F. Prins, D. K. Kim, J. Cui et al., "Direct patterning of colloidal quantum-dot thin films for enhanced and spectrally selective out-coupling of emission," Nano Letters, vol. 17, no. 3, pp. 1319-1325, 2017.

[19] S. Mladenovski, K. Neyts, D. Pavicic, A. Werner, and C. Rothe, "Exceptionally efficient organic light emitting devices using high refractive index substrates," Optics Express, vol. 17, no. 9, pp. 7562-7570, 2009.

[20] H. Liang, R. Zhu, Y. Dong et al., "Enhancing the outcoupling efficiency of quantum dot LEDs with internal nano-scattering pattern," Optics Express, vol. 23, no. 10, pp. 12910-12922, 2015. 
[21] Y. R. Do, Y. C. Kim, Y. W. Song et al., "Enhanced light extraction from organic light-emitting diodes with $2 \mathrm{D} \mathrm{Sio}_{2} / \mathrm{Sin}_{\mathrm{x}}$ photonic crystals," Advanced Materials, vol. 15, no. 14, pp. 1214-1218, 2003.

[22] S. Möller and S. R. Forrest, "Improved light out-coupling in organic light emitting diodes employing ordered microlens arrays," Journal of Applied Physics, vol. 91, no. 5, pp. 33243327, 2002.

[23] C.-L. Lin, T.-Y. Cho, C.-H. Chang, and C.-C. Wu, "Enhancing light outcoupling of organic light-emitting devices by locating emitters around the second antinode of the reflective metal electrode," Applied Physics Letters, vol. 88, no. 8, article 081114, 2006.

[24] D. Yokoyama, "Molecular orientation in small-molecule organic light-emitting diodes," Journal of Materials Chemistry, vol. 21, no. 48, pp. 19187-19202, 2011.

[25] J. Frischeisen, D. Yokoyama, C. Adachi, and W. Brütting, "Determination of molecular dipole orientation in doped fluorescent organic thin films by photoluminescence measurements," Applied Physics Letters, vol. 96, no. 7, article 073302, 2010.

[26] S. Ithurria, M. D. Tessier, B. Mahler, R. P. S. M. Lobo, B. Dubertret, and A. L. Efros, "Colloidal nanoplatelets with two-dimensional electronic structure," Nature Materials, vol. 10, no. 12, pp. 936-941, 2011.

[27] D. V. Talapin, J.-S. Lee, M. V. Kovalenko, and E. V. Shevchenko, "Prospects of colloidal nanocrystals for electronic and optoelectronic applications," Chemical Reviews, vol. 110, no. 1, pp. 389-458, 2010.

[28] M. D. Tessier, P. Spinicelli, D. Dupont, G. Patriarche, S. Ithurria, and B. Dubertret, "Efficient exciton concentrators built from colloidal core/crown CdSe/CdS semiconductor nanoplatelets," Nano Letters, vol. 14, no. 1, pp. 207-213, 2014.

[29] M. D. Tessier, C. Javaux, I. Maksimovic, V. Loriette, and B. Dubertret, "Spectroscopy of single CdSe nanoplatelets," ACS Nano, vol. 6, no. 8, pp. 6751-6758, 2012.

[30] B. Mahler, B. Nadal, C. Bouet, G. Patriarche, and B. Dubertret, "Core/shell colloidal semiconductor nanoplatelets," Journal of the American Chemical Society, vol. 134, no. 45, pp. 18591-18598, 2012.

[31] H. Htoon, J. A. Hollingsworth, R. Dickerson, and V. I. Klimov, "Effect of zero- to one-dimensional transformation on multiparticle Auger recombination in semiconductor quantum rods," Physical Review Letters, vol. 91, no. 22, p. 227401, 2003.

[32] H. Zhu and T. Lian, "Enhanced multiple exciton dissociation from CdSe quantum rods: the effect of nanocrystal shape," Journal of the American Chemical Society, vol. 134, no. 27, pp. 11289-11297, 2012.

[33] Q. Li and T. Lian, "Area- and thickness-dependent biexciton Auger recombination in colloidal CdSe nanoplatelets: breaking the "universal volume scaling law"," Nano Letters, vol. 17, no. 5, pp. 3152-3158, 2017.

[34] S.-F. Wu, S.-H. Li, Y.-K. Wang et al., "White organic LED

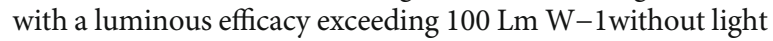
out-coupling enhancement techniques," Advanced Functional Materials, vol. 27, no. 31, p. 1701314, 2017.

[35] J.-H. Jou, C. Y. Hsieh, J. R. Tseng et al., "Candle light-style organic light-emitting diodes," Advanced Functional Materials, vol. 23, no. 21, pp. 2750-2757, 2013.
[36] B. Liu, L. Wang, D. Gao et al., "Harnessing charge and exciton distribution towards extremely high performance: the critical role of guests in single-emitting-layer white OLEDs," Materials Horizons, vol. 2, no. 5, pp. 536-544, 2015.

[37] B. Liu, J. Zou, Z. Zhou et al., "Efficient single-emitting layer hybrid white organic light-emitting diodes with low efficiency roll-off, stable color and extremely high luminance," Journal of Industrial and Engineering Chemistry, vol. 30, pp. 85-91, 2015.

[38] B. Liu, S. Delikanli, Y. Gao, D. Dede, K. Gungor, and H. V. Demir, "Nanocrystal light-emitting diodes based on type II nanoplatelets," Nano Energy, vol. 47, pp. 115-122, 2018.

[39] D. Kim, Y. Fu, S. Kim et al., "Polyethylenimine ethoxylatedmediated all-solution-processed high-performance flexible inverted quantum dot-light-emitting device," ACS Nano, vol. 11, no. 2, pp. 1982-1990, 2017.

[40] H. Shen, Q. Lin, W. Cao et al., "Efficient and long-lifetime full-color light-emitting diodes using high luminescence quantum yield thick-shell quantum dots," Nanoscale, vol. 9, no. 36, pp. 13583-13591, 2017.

[41] K.-H. Lee, J. H. Lee, H. D. Kang et al., “Over 40 Cd/a efficient green quantum dot electroluminescent device comprising uniquely large-sized quantum dots," ACS Nano, vol. 8, no. 5, pp. 4893-4901, 2014.

[42] U. Giovanella, M. Pasini, M. Lorenzon et al., "Efficient solution-processed nanoplatelet-based light-emitting diodes with high operational stability in air," Nano Letters, vol. 18, no. 6, pp. 3441-3448, 2018.

[43] J. Lim, B. G. Jeong, M. Park et al., "Influence of shell thickness on the performance of light-emitting devices based on CdSe/ $\mathrm{Zn}_{1-\mathrm{X}} \mathrm{cd}_{\mathrm{x}} \mathrm{s}$ core/shell heterostructured quantum dots," Advanced Materials, vol. 26, no. 47, pp. 8034-8040, 2014.

[44] B. S. Mashford, M. Stevenson, Z. Popovic et al., "High-efficiency quantum-dot light-emitting devices with enhanced charge injection," Nature Photonics, vol. 7, no. 5, pp. 407412, 2013.

[45] S. Coe, W. K. Woo, M. Bawendi, and V. Bulović, "Electroluminescence from single monolayers of nanocrystals in molecular organic devices," Nature, vol. 420, no. 6917, pp. 800-803, 2002.

[46] T. Higuchi, H. Nakanotani, and C. Adachi, "High-efficiency white organic light-emitting diodes based on a blue thermally activated delayed fluorescent emitter combined with green and red fluorescent emitters," Advanced Materials, vol. 27, no. 12, pp. 2019-2023, 2015.

[47] F. Liang, Y. Liu, Y. Hu et al., "Polymer as an additive in the emitting layer for high-performance quantum dot lightemitting diodes," ACS Applied Materials \& Interfaces, vol. 9, no. 23, pp. 20239-20246, 2017.

[48] O. Heikkilä, J. Oksanen, and J. Tulkki, "Ultimate limit and temperature dependency of light-emitting diode efficiency," Journal of Applied Physics, vol. 105, no. 9, article 093119, 2009.

[49] S.-J. Su, E. Gonmori, H. Sasabe, and J. Kido, "Highly efficient organic blue-and white-light-emitting devices having a carrierand exciton-confining structure for reduced efficiency roll-off," Advanced Materials, vol. 20, no. 21, pp. 4189-4194, 2008.

[50] H. Choi, B. S. Kim, M. J. Ko et al., "Solution processed $\mathrm{Wo}_{3}$ layer for the replacement of PEDOT:PSS layer in organic photovoltaic cells," Organic Electronics, vol. 13, no. 6, pp. 959-968, 2012. 
[51] Z. Luo, Y. Chen, and S. T. Wu, "Wide color gamut LCD with a quantum dot backlight," Optics Express, vol. 21, no. 22, pp. 26269-26284, 2013.

[52] Z. A. Peng and X. Peng, "Nearly monodisperse and shapecontrolled CdSe nanocrystals via alternative routes: nucleation and growth," Journal of the American Chemical Society, vol. 124, no. 13, pp. 3343-3353, 2002.

[53] S. Kumar and T. Nann, "Shape control of II-VI semiconductor nanomaterials," Small, vol. 2, no. 3, pp. 316-329, 2006.

[54] S. Ithurria and B. Dubertret, "Quasi 2D colloidal CdSe platelets with thicknesses controlled at the atomic level," Journal of the American Chemical Society, vol. 130, no. 49, pp. 16504-16505, 2008.

[55] S. Ithurria, G. Bousquet, and B. Dubertret, "Continuous transition from $3 \mathrm{D}$ to $1 \mathrm{D}$ confinement observed during the formation of CdSe nanoplatelets," Journal of the American Chemical Society, vol. 133, no. 9, pp. 3070-3077, 2011.

[56] Y. Chen, D. Chen, Z. Li, and X. Peng, "Symmetry-breaking for formation of rectangular CdSe two-dimensional nanocrystals in zinc-blende structure," Journal of the American Chemical Society, vol. 139, no. 29, pp. 10009-10019, 2017.

[57] F. D. Ott, A. Riedinger, D. R. Ochsenbein et al., "Ripening of semiconductor nanoplatelets," Nano Letters, vol. 17, no. 11, pp. 6870-6877, 2017.

[58] S. Ithurria and D. V. Talapin, "Colloidal atomic layer deposition (C-Ald) using self-limiting reactions at nanocrystal surface coupled to phase transfer between polar and nonpolar media," Journal of the American Chemical Society, vol. 134, no. 45, pp. 18585-18590, 2012.

[59] S. Pedetti, S. Ithurria, H. Heuclin, G. Patriarche, and B. Dubertret, "Type-II CdSe/CdTe core/crown semiconductor nanoplatelets," Journal of the American Chemical Society, vol. 136, no. 46, pp. 16430-16438, 2014.

[60] P. Tyagi, S. M. Arveson, and W. A. Tisdale, "Colloidal organohalide perovskite nanoplatelets exhibiting quantum confinement," Journal of Physical Chemistry Letters, vol. 6, no. 10, pp. 1911-1916, 2015.

[61] Y. Bekenstein, B. A. Koscher, S. W. Eaton, P. Yang, and A. P. Alivisatos, "Highly luminescent colloidal nanoplates of perovskite cesium lead halide and their oriented assemblies," Journal of the American Chemical Society, vol. 137, no. 51, pp. 16008-16011, 2015.

[62] Q. A. Akkerman, S. G. Motti, A. R. Srimath Kandada et al., "Solution synthesis approach to colloidal cesium lead halide perovskite nanoplatelets with monolayer-level thickness control," Journal of the American Chemical Society, vol. 138, no. 3, pp. 1010-1016, 2016.

[63] G. H. V. Bertrand, A. Polovitsyn, S. Christodoulou, A. H. Khan, and I. Moreels, "Shape control of zincblende CdSe nanoplatelets," Chemical Communications, vol. 52, no. 80, pp. 11975-11978, 2016.

[64] A. Riedinger, F. D. Ott, A. Mule et al., "An intrinsic growth instability in isotropic materials leads to quasi-two- dimensional nanoplatelets," Nature Materials, vol. 16, no. 7, pp. 743-748, 2017.

[65] S. Christodoulou, J. I. Climente, J. Planelles et al., "Chlorideinduced thickness control in CdSe nanoplatelets," Nano Letters, vol. 18, no. 10, pp. 6248-6254, 2018.

[66] W. Cho, S. Kim, I. Coropceanu et al., "Direct synthesis of sixmonolayer $(1.9 \mathrm{Nm})$ thick zinc-blende CdSe nanoplatelets emitting at $585 \mathrm{Nm}$," Chemistry of Materials, vol. 30, no. 20 , pp. $6957-6960,2018$.

[67] J. Zhao, S. Cao, Z. Li, and N. Ma, “Amino acid-mediated synthesis of $\mathrm{CsPbBr}_{3}$ Perovskite nanoplatelets with tunable thickness and optical properties," Chemistry of Materials, vol. 30, no. 19, pp. 6737-6743, 2018.

[68] M. C. Weidman, M. Seitz, S. D. Stranks, and W. A. Tisdale, "Highly tunable colloidal perovskite nanoplatelets through variable cation, metal, and halide composition," ACS Nano, vol. 10, no. 8, pp. 7830-7839, 2016.

[69] J. Lim, W. K. Bae, K. U. Park et al., "Controlled synthesis of CdSe tetrapods with high morphological uniformity by the persistent kinetic growth and the halide-mediated phase transformation," Chemistry of Materials, vol. 25, no. 8, pp. 1443-1449, 2013.

[70] M. Meyns, F. Iacono, C. Palencia et al., "Shape evolution of CdSe nanoparticles controlled by halogen compounds," Chemistry of Materials, vol. 26, no. 5, pp. 1813-1821, 2014.

[71] F. Gerdes, C. Navío, B. H. Juárez, and C. Klinke, "Size, shape, and phase control in ultrathin CdSe nanosheets," Nano Letters, vol. 17, no. 7, pp. 4165-4171, 2017.

[72] Z. Liang, S. Zhao, Z. Xu et al., "Shape-controlled synthesis of all-inorganic $\mathrm{CsPbBr} 3$ Perovskite nanocrystals with bright blue emission," ACS Applied Materials \& Interfaces, vol. 8, no. 42, pp. 28824-28830, 2016.

[73] P. Michler, A. Imamoğlu, M. D. Mason, P. J. Carson, G. F. Strouse, and S. K. Buratto, "Quantum correlation among photons from a single quantum dot at room temperature," Nature, vol. 406, no. 6799, pp. 968-970, 2000.

[74] O. Chen, J. Zhao, V. P. Chauhan et al., "Compact high-quality CdSe-CdS core-shell nanocrystals with narrow emission linewidths and suppressed blinking," Nature Materials, vol. 12, no. 5, pp. 445-451, 2013.

[75] K. Yu, B. Zaman, S. Romanova, D. S. Wang, and J. A. Ripmeester, "Sequential synthesis of type II colloidal CdTe/CdSe core-shell nanocrystals," Small, vol. 1, no. 3, pp. 332-338, 2005.

[76] A. Polovitsyn, Z. Dang, J. L. Movilla et al., "Synthesis of airstable CdSe/ZnS core-shell nanoplatelets with tunable emission wavelength," Chemistry of Materials, vol. 29, no. 13, pp. 5671-5680, 2017.

[77] S. J. Oh, N. E. Berry, J. H. Choi et al., "Stoichiometric control of lead chalcogenide nanocrystal solids to enhance their electronic and optoelectronic device performance," ACS Nano, vol. 7, no. 3, pp. 2413-2421, 2013.

[78] M. D. Tessier, B. Mahler, B. Nadal, H. Heuclin, S. Pedetti, and B. Dubertret, "Spectroscopy of colloidal semiconductor core/ shell nanoplatelets with high quantum yield," Nano Letters, vol. 13, no. 7, pp. 3321-3328, 2013.

[79] A. A. Rossinelli, A. Riedinger, P. Marqués-Gallego, P. N. Knüsel, F. V. Antolinez, and D. J. Norris, "High-temperature growth of thick-shell CdSe/CdS core/shell nanoplatelets," Chemical Communications, vol. 53, no. 71, pp. 9938-9941, 2017.

[80] Y. Altintas, U. Quliyeva, K. Gungor et al., "Highly stable, near-unity efficiency atomically flat semiconductor nanocrystals of CdSe/ZnS hetero-nanoplatelets enabled by ZnSshell hot-injection growth," Small, vol. 15, no. 8, p. 1804854, 2019.

[81] A. A. Rossinelli, H. Rojo, A. S. Mule et al., "Compositional grading for efficient and narrowband emission in CdSe- 
based core/shell nanoplatelets," Chemistry of Materials, vol. 31, no. 22, pp. 9567-9578, 2019.

[82] A. H. Khan, G. H. V. Bertrand, A. Teitelboim et al., "CdSe/ CdS/CdTe core/barrier/crown nanoplatelets: synthesis, optoelectronic properties, and multiphoton fluorescence upconversion," ACS Nano, vol. 14, no. 4, pp. 4206-4215, 2020.

[83] M. Sharma, M. Olutas, A. Yeltik et al., "Understanding the journey of dopant copper ions in atomically flat colloidal nanocrystals of CdSe nanoplatelets using partial cation exchange reactions," Chemistry of Materials, vol. 30, no. 10, pp. 3265-3275, 2018.

[84] M. Gopal, "Ag and Cu doped colloidal CdSe nanocrystals: partial cation exchange and luminescence," Materials Research Express, vol. 2, no. 8, article 085004, 2015.

[85] M. Dufour, E. Izquierdo, C. Livache et al., "Doping as a strategy to tune color of 2D colloidal nanoplatelets," ACS Applied Materials \& Interfaces, vol. 11, no. 10, pp. 10128-10134, 2019.

[86] A. H. Khan, V. Pinchetti, I. Tanghe et al., "Tunable and efficient red to near-infrared photoluminescence by synergistic exploitation of core and surface silver doping of CdSe nanoplatelets," Chemistry of Materials, vol. 31, no. 4, pp. 1450-1459, 2019.

[87] T. Galle, M. Kazes, R. Hübner et al., "Colloidal mercurydoped CdSe nanoplatelets with dual fluorescence," Chemistry of Materials, vol. 31, no. 14, pp. 5065-5074, 2019.

[88] S. Delikanli, M. Z. Akgul, J. R. Murphy et al., "Mn2+-doped $\mathrm{CdSe} / \mathrm{CdS}$ core/multishell colloidal quantum wells enabling tunable carrier dopant exchange interactions," ACS Nano, vol. 9, no. 12, pp. 12473-12479, 2015.

[89] W. J. Mir, M. Jagadeeswararao, S. Das, and A. Nag, "Colloidal Mn-doped cesium lead halide perovskite nanoplatelets," ACS Energy Letters, vol. 2, no. 3, pp. 537-543, 2017.

[90] Z.-J. Li, E. Hofman, A. H. Davis et al., "Complete dopant substitution by spinodal decomposition in Mn-doped twodimensional CsPbCl3Nanoplatelets," Chemistry of Materials, vol. 30, no. 18, pp. 6400-6409, 2018.

[91] A. H. Davis, S. Li, H. Lin et al., "Ligand-mediated synthesis of chemically tailored two-dimensional all-inorganic perovskite nanoplatelets under ambient conditions," Journal of Materials Chemistry C, vol. 9, no. 40, pp. 14226-14235, 2021.

[92] K. Justice Babu, G. Kaur, A. Shukla et al., "Concurrent energy- and electron-transfer dynamics in photoexcited Mn-doped $\mathrm{CsPbBr}_{3}$ Perovskite nanoplatelet architecture," Journal of Physical Chemistry Letters, vol. 12, no. 1, pp. 302-309, 2021.

[93] A. G. Vitukhnovsky, V. S. Lebedev, A. S. Selyukov, A. A. Vashchenko, R. B. Vasiliev, and M. S. Sokolikova, "Electroluminescence from colloidal semiconductor CdSe nanoplatelets in hybrid organic-inorganic light emitting diode," Chemical Physics Letters, vol. 619, pp. 185-188, 2015.

[94] Y. Kelestemur, Y. Shynkarenko, M. Anni, S. Yakunin, M. L. de Giorgi, and M. V. Kovalenko, "Colloidal CdSe quantum wells with graded shell composition for low-threshold amplified spontaneous emission and highly efficient electroluminescence," ACS Nano, vol. 13, no. 12, pp. 13899-13909, 2019.

[95] B. Liu, Y. Altintas, L. Wang et al., "Record high external quantum efficiency of $19.2 \%$ achieved in light-emitting diodes of colloidal quantum wells enabled by hot-injection shell growth," Advanced Materials, vol. 32, no. 8, p. 1905824, 2020.

[96] B. Liu, X. L. Li, H. Tao et al., "Manipulation of exciton distribution for high-performance fluorescent/phosphorescent hybrid white organic light-emitting diodes," Journal of Materials Chemistry C, vol. 5, no. 31, pp. 7668-7683, 2017.

[97] W. Qin, J. Liu, S. Chen et al., “Crafting NPB with tetraphenylethene: a win-win strategy to create stable and efficient solidstate emitters with aggregation-induced emission feature, high hole-transporting property and efficient electroluminescence," Journal of Materials Chemistry C, vol. 2, no. 19, pp. 3756-3761, 2014.

[98] F. Zhang, S. Wang, L. Wang et al., "Super color purity green quantum dot light-emitting diodes fabricated by using CdSe/CdS nanoplatelets," Nanoscale, vol. 8, no. 24, pp. 12182-12188, 2016.

[99] C. She, I. Fedin, D. S. Dolzhnikov et al., "Low-threshold stimulated emission using colloidal quantum wells," Nano Letters, vol. 14, no. 5, pp. 2772-2777, 2014.

[100] M. Olutas, B. Guzelturk, Y. Kelestemur, A. Yeltik, S. Delikanli, and H. V. Demir, "Lateral size-dependent spontaneous and stimulated emission properties in colloidal CdSe nanoplatelets," ACS Nano, vol. 9, no. 5, pp. 5041-5050, 2015.

[101] K. Wu, Q. Li, Y. Jia, J. R. McBride, Z. X. Xie, and T. Lian, "Efficient and ultrafast formation of long-lived chargetransfer exciton state in atomically thin cadmium selenide/ cadmium telluride type-II heteronanosheets," ACS Nano, vol. 9, no. 1, pp. 961-968, 2015.

[102] Y. Yang, Y. Zheng, W. Cao et al., "High-efficiency lightemitting devices based on quantum dots with tailored nanostructures," Nature Photonics, vol. 9, no. 4, pp. 259266, 2015.

[103] H. Zhang, H. Li, X. Sun, and S. Chen, "Inverted quantum-dot light-emitting diodes fabricated by all-solution processing," ACS Applied Materials \& Interfaces, vol. 8, no. 8, pp. 54935498, 2016.

[104] Z. Wen, P. Liu, J. Ma et al., "High-performance ultrapure green $\mathrm{CdSe} / \mathrm{CdS}$ core/crown nanoplatelet light-emitting diodes by suppressing nonradiative energy transfer," Advanced Electronic Materials, vol. 7, no. 7, p. 2000965, 2021.

[105] Z. Wen, C. Zhang, Z. Zhou et al., "Ultrapure green lightemitting diodes based on CdSe/CdS core/crown nanoplatelets," IEEE Journal of Quantum Electronics, vol. 56, no. 1, pp. 1-6, 2020.

[106] Z. Li, H. Qin, D. Guzun, M. Benamara, G. Salamo, and X. Peng, "Uniform thickness and colloidal-stable CdS quantum disks with tunable thickness: synthesis and properties," Nano Research, vol. 5, no. 5, pp. 337-351, 2012.

[107] Y. Gao, M. Li, S. Delikanli et al., "Low-threshold lasing from colloidal CdSe/CdSete core/alloyed-crown type-II heteronanoplatelets," Nanoscale, vol. 10, no. 20, pp. 94669475, 2018.

[108] M. M. Lee, J. Teuscher, T. Miyasaka, T. N. Murakami, and H. J. Snaith, "Efficient hybrid solar cells based on mesosuperstructured organometal halide perovskites," Science, vol. 338, no. 6107, pp. 643-647, 2012.

[109] L. Protesescu, S. Yakunin, M. I. Bodnarchuk et al., "Nanocrystals of cesium lead halide perovskites $\left(\mathrm{CsPbX}_{3}, \mathrm{X}=\mathrm{Cl}\right.$, $\mathrm{Br}$, and I): novel optoelectronic materials showing bright emission with wide color gamut," Nano Letters, vol. 15, no. 6, pp. 3692-3696, 2015.

[110] S. Yakunin, M. Sytnyk, D. Kriegner et al., "Detection of X-ray photons by solution-processed lead halide perovskites," Nature Photonics, vol. 9, no. 7, pp. 444-449, 2015. 
[111] J. Luo, J. H. Im, M. T. Mayer et al., "Water photolysis at $12.3 \%$ efficiency via perovskite photovoltaics and earth-abundant catalysts," Science, vol. 345, no. 6204, pp. 1593-1596, 2014.

[112] H. Zhu, Y. Fu, F. Meng et al., "Lead halide perovskite nanowire lasers with low lasing thresholds and high quality factors," Nature Materials, vol. 14, no. 6, pp. 636-642, 2015.

[113] B. A. Koscher, J. K. Swabeck, N. D. Bronstein, and A. P. Alivisatos, "Essentially trap-free $\mathrm{CsPbBr} 3$ Colloidal nanocrystals by postsynthetic thiocyanate surface treatment," Journal of the American Chemical Society, vol. 139, no. 19, pp. 65666569, 2017.

[114] A. Swarnkar, R. Chulliyil, V. K. Ravi, M. Irfanullah, A. Chowdhury, and A. Nag, "Colloidal $\mathrm{CsPbr}_{3}$ perovskite nanocrystals: luminescence beyond traditional quantum dots," Angewandte Chemie, International Edition, vol. 54, no. 51, pp. 15424-15428, 2015.

[115] Y. Zhang, J. Liu, Z. Wang et al., "Synthesis, properties, and optical applications of low-dimensional perovskites," Chemical Communications, vol. 52, no. 94, pp. 13637-13655, 2016.

[116] J. Song, J. Li, X. Li, L. Xu, Y. Dong, and H. Zeng, "Quantum dot light-emitting diodes based on inorganic perovskite cesium lead halides $\left(\mathrm{CsPbX}_{3}\right)$," Advanced Materials, vol. 27, no. 44, pp. 7162-7167, 2015.

[117] Z. K. Tan, R. S. Moghaddam, M. L. Lai et al., "Bright lightemitting diodes based on organometal halide perovskite," Nature Nanotechnology, vol. 9, no. 9, pp. 687-692, 2014.

[118] Y.-H. Kim, H. Cho, J. H. Heo et al., "Multicolored organic/ inorganic hybrid perovskite light-emitting diodes," Advanced Materials, vol. 27, no. 7, pp. 1248-1254, 2015.

[119] J. Wang, N. Wang, Y. Jin et al., "Interfacial control toward efficient and low-voltage perovskite light-emitting diodes," Advanced Materials, vol. 27, no. 14, pp. 2311-2316, 2015.

[120] Y. Ling, Z. Yuan, Y. Tian et al., "Bright light-emitting diodes based on organometal halide perovskite nanoplatelets," Advanced Materials, vol. 28, no. 2, pp. 305-311, 2016.

[121] G. Li, F. W. R. Rivarola, N. J. L. K. Davis et al., "Highly efficient perovskite nanocrystal light-emitting diodes enabled by a universal crosslinking method," Advanced Materials, vol. 28, no. 18, pp. 3528-3534, 2016.

[122] G. Li, Z. K. Tan, D. Di et al., "Efficient light-emitting diodes based on nanocrystalline perovskite in a dielectric polymer matrix," Nano Letters, vol. 15, no. 4, pp. 26402644, 2015.

[123] X. Zhang, H. Lin, H. Huang et al., "Enhancing the brightness of cesium lead halide perovskite nanocrystal based green light-emitting devices through the interface engineering with perfluorinated ionomer," Nano Letters, vol. 16, no. 2, pp. 1415-1420, 2016.

[124] N. K. Kumawat, A. Dey, A. Kumar, S. P. Gopinathan, K. L. Narasimhan, and D. Kabra, "Band gap tuning of $\mathrm{CH}_{3} \mathrm{NH}_{3} \mathrm{~Pb}\left(\mathrm{Br}_{1-x} \mathrm{Cl}_{x}\right)_{3}$ Hybrid perovskite for blue electroluminescence," ACS Applied Materials \& Interfaces, vol. 7, no. 24, pp. 13119-13124, 2015.

[125] S. D. Stranks, G. E. Eperon, G. Grancini et al., "Electron-hole diffusion lengths exceeding 1 micrometer in an organometal trihalide perovskite absorber," Science, vol. 342, no. 6156, pp. 341-344, 2013.

[126] G. Xing, N. Mathews, S. Sun et al., "Long-range balanced electron- and hole-transport lengths in organic-inorganic $\mathrm{CH}_{3} \mathrm{NH}_{3} \mathrm{PbI}_{3}$," Science, vol. 342, no. 6156, pp. 344-347, 2013.
[127] X. Zhang, H. Liu, W. Wang et al., "Hybrid perovskite lightemitting diodes based on perovskite nanocrystals with organic-inorganic mixed cations," Advanced Materials, vol. 29, no. 18, p. 1606405, 2017.

[128] Q. Dong, Y. Fang, Y. Shao et al., "Electron-hole diffusion lengths $>175 \mu \mathrm{m}$ in solution-grown $\mathrm{CH}_{3} \mathrm{NH}_{3} \mathrm{PbI}_{3}$ single crystals," Science, vol. 347, no. 6225, pp. 967-970, 2015.

[129] Y. Fang, Q. Dong, Y. Shao, Y. Yuan, and J. Huang, "Highly narrowband perovskite single-crystal photodetectors enabled by surface-charge recombination," Nature Photonics, vol. 9, no. 10, pp. 679-686, 2015.

[130] D. Liang, Y. Peng, Y. Fu et al., "Color-pure violet-lightemitting diodes based on layered lead halide perovskite nanoplates," ACS Nano, vol. 10, no. 7, pp. 6897-6904, 2016.

[131] S. Kumar, J. Jagielski, S. Yakunin et al., "Efficient blue electroluminescence using quantum-confined two-dimensional perovskites," ACS Nano, vol. 10, no. 10, pp. 9720-9729, 2016.

[132] S. Kumar, J. Jagielski, N. Kallikounis et al., "Ultrapure green light-emitting diodes using two-dimensional formamidinium perovskites: achieving recommendation 2020 color coordinates," Nano Letters, vol. 17, no. 9, pp. 52775284, 2017.

[133] C. Qin, T. Matsushima, T. Fujihara, W. J. Potscavage Jr., and C. Adachi, "Degradation mechanisms of solution-processed planar perovskite solar cells: thermally stimulated current measurement for analysis of carrier traps," Advanced Materials, vol. 28, no. 3, pp. 466-471, 2016.

[134] N.-G. Park, M. Grätzel, T. Miyasaka, K. Zhu, and K. Emery, "Towards stable and commercially available perovskite solar cells," Nature Energy, vol. 1, no. 11, p. 16152, 2016.

[135] G. R. Yettapu, D. Talukdar, S. Sarkar et al., "Terahertz conductivity within colloidal $\mathrm{Cs} \mathrm{PbBr}_{3}$ perovskite nanocrystals: remarkably high carrier mobilities and large diffusion lengths," Nano Letters, vol. 16, no. 8, pp. 4838-4848, 2016.

[136] N. Yantara, S. Bhaumik, F. Yan et al., "Inorganic halide perovskites for efficient light-emitting diodes," Journal of Physical Chemistry Letters, vol. 6, no. 21, pp. 4360-4364, 2015.

[137] S. Yakunin, L. Protesescu, F. Krieg et al., "Low-threshold amplified spontaneous emission and lasing from colloidal nanocrystals of caesium lead halide perovskites," Nature Communications, vol. 6, no. 1, p. 8056, 2015.

[138] E. Yassitepe, Z. Yang, O. Voznyy et al., “Amine-free synthesis of cesium lead halide perovskite quantum dots for efficient light-emitting diodes," Advanced Functional Materials, vol. 26, no. 47, pp. 8757-8763, 2016.

[139] L. Zhang, X. Yang, Q. Jiang et al., "Ultra-bright and highly efficient inorganic based perovskite light-emitting diodes," Nature Communications, vol. 8, no. 1, p. 15640, 2017.

[140] X. Zhang, B. Xu, J. Zhang et al., "All-inorganic perovskite nanocrystals for high-efficiency light emitting diodes: dualphase $\mathrm{Cs} \mathrm{PbBr}_{3}-\mathrm{CsPb}_{2} \mathrm{Br}_{5}$ Composites," Advanced Functional Materials, vol. 26, no. 25, pp. 4595-4600, 2016.

[141] C. Qin, T. Matsushima, A. S. D. Sandanayaka, Y. Tsuchiya, and C. Adachi, "Centrifugal-coated quasi-two-dimensional perovskite $\mathrm{CsPb}_{2} \mathrm{Br}_{5}$ Films for efficient and stable lightemitting diodes," Journal of Physical Chemistry Letters, vol. 8, no. 21, pp. 5415-5421, 2017.

[142] C. Han, C. Li, Z. Zang et al., "Tunable luminescent $\mathrm{CsPb}_{2} \mathrm{Br}_{5}$ nanoplatelets: applications in light-emitting diodes and 
photodetectors," Photonics Research, vol. 5, no. 5, pp. 473480, 2017.

[143] J. Si, Y. Liu, Z. He et al., "Efficient and high-color-purity lightemitting diodes based onIn SituGrown films of $\mathrm{CsPbX} 3(\mathrm{X}=$ $\mathrm{Br}, \mathrm{I})$ nanoplates with controlled thicknesses," ACS Nano, vol. 11, no. 11, pp. 11100-11107, 2017.

[144] Y. Tian, C. Zhou, M. Worku et al., "Highly efficient spectrally stable red perovskite light-emitting diodes," Advanced Materials, vol. 30, no. 20, p. 1707093, 2018.

[145] D. Yang, Y. Zou, P. Li et al., "Large-scale synthesis of ultrathin cesium lead bromide perovskite nanoplates with precisely tunable dimensions and their application in blue light-emitting diodes," Nano Energy, vol. 47, pp. 235-242, 2018.

[146] R. L. Z. Hoye, M. L. Lai, M. Anaya et al., "Identifying and reducing interfacial losses to enhance color-pure electroluminescence in blue-emitting perovskite nanoplatelet lightemitting diodes," ACS Energy Letters, vol. 4, no. 5, pp. 1181-1188, 2019.

[147] C. Zhang, Q. Wan, B. Wang et al., "Surface ligand engineering toward brightly luminescent and stable cesium lead halide perovskite nanoplatelets for efficient blue-light-emitting diodes," Journal of Physical Chemistry C, vol. 123, no. 43, pp. 26161-26169, 2019.

[148] R. Scott, J. Heckmann, A. V. Prudnikau et al., "Directed emission of CdSe nanoplatelets originating from strongly anisotropic 2D electronic structure," Nature Nanotechnology, vol. 12, no. 12, pp. 1155-1160, 2017.

[149] Y. Gao, M. C. Weidman, and W. A. Tisdale, "CdSe nanoplatelet films with controlled orientation of their transition dipole moment," Nano Letters, vol. 17, no. 6, pp. 38373843, 2017.

[150] R. Momper, H. Zhang, S. Chen et al., "Kinetic control over self-assembly of semiconductor nanoplatelets," Nano Letters, vol. 20, no. 6, pp. 4102-4110, 2020.

[151] P. Bai, A. Hu, Y. Liu, Y. Jin, and Y. Gao, "Printing andIn SituAssembly of CdSe/CdS nanoplatelets as uniform films with unity in-plane transition dipole moment," Journal of Physical Chemistry Letters, vol. 11, no. 11, pp. 4524-4529, 2020.

[152] K. Sudhir, M. Tommaso, K. Frank, L. Yen-Ting, C. YuCheng, and S. Chih-Jen, "Two-dimensional nanoplatelet superlattices overcoming light outcoupling efficiency limit in perovskite quantum dot light-emitting diodes," Research Square, 2021.

[153] J. Cui, Y. Liu, Y. Deng et al., "Efficient light-emitting diodes based on oriented perovskite nanoplatelets," Science Advances, vol. 7, no. 41, article eabg8458, 2021.

[154] B. Liu, H. Nie, G. Lin et al., "High-performance doping-free hybrid white OLEDs based on blue aggregation-induced emission luminogens," ACS Applied Materials \& Interfaces, vol. 9, no. 39, pp. 34162-34171, 2017.

[155] B. Liu, D. Luo, J. Zou et al., "A host-guest system comprising high guest concentration to achieve simplified and highperformance hybrid white organic light-emitting diodes," Journal of Materials Chemistry C, vol. 3, no. 24, pp. 63596366, 2015.

[156] N. Sun, Q. Wang, Y. Zhao et al., "A hybrid white organic light-emitting diode with above $20 \%$ external quantum efficiency and extremely low efficiency roll-off," Journal of Materials Chemistry C, vol. 2, no. 36, pp. 7494-7504, 2014.
[157] D. Luo, Y. Xiao, M. Hao et al., "Doping-free white organic light-emitting diodes without blue molecular emitter: an unexplored approach to achieve high performance via exciplex emission," Applied Physics Letters, vol. 110, no. 6, article 061105, 2017.

[158] L. Duan, D. Zhang, K. Wu, X. Huang, L. Wang, and Y. Qiu, "Controlling the recombination zone of white organic lightemitting diodes with extremely long lifetimes," Advanced Functional Materials, vol. 21, no. 18, pp. 3540-3545, 2011. 